\title{
Hunting the Flavon
}

\author{
Martin Bauer, ${ }^{1}$ Torben Schell, ${ }^{1}$ and Tilman Plehn ${ }^{1}$ \\ ${ }^{1}$ Institut für Theoretische Physik, Universität Heidelberg, Germany
}

(Dated: March 24, 2016)

The next generation of experiments in particle physics will for the first time systematically test flavor physics models based on flavon fields. Starting from the current quark-flavor constrains on such models we show how the new generation of lepton flavor experiments will dominate indirect searches in the coming decades. A future $100 \mathrm{TeV}$ hadron collider will then be the first experiment to probe flavons as propagating degrees of freedom. Our estimate of the collider reach relies on a proper treatment of backgrounds and detector effects. Complementary searches for indirect effects in lepton flavor experiments and propagating degrees of freedom at colliders are very limited at the LHC, but will be a new feature at a $100 \mathrm{TeV}$ hadron collider. 


\section{CONTENTS}

I. Introduction

II. Flavon model

Flavon couplings

Flavon and top decays 6

\begin{tabular}{lr} 
III. Quark flavor constraints & 7 \\
\hline
\end{tabular}

Neutral meson mixing

Leptonic meson decays $\quad 9$

IV. Future lepton flavor measurements 11

Decay $\mu \rightarrow e \gamma$

Conversion $\mu \rightarrow e \quad 12$

Decays $\mu \rightarrow 3 e$ and $\tau \rightarrow 3 \mu$

V. Future hadron collider measurements 14

Resonance searches

Associated production

Same-sign top pairs

\begin{tabular}{ll} 
Flavon pair production & 19 \\
\hline
\end{tabular}

\begin{tabular}{lr} 
VI. Outlook & 19 \\
\hline
\end{tabular}

Acknowledgments

A. Benchmark Point 21

References 


\section{INTRODUCTION}

The structure of the quark and lepton flavor sectors is one of the biggest mysteries of particle physics. Various extensions of the Standard Model (SM) address the flavor structure for example through abelian flavor symmetries [1, 2], loop-suppressed couplings to the Higgs [3, partial compositeness [4, or wave-function localization [5]. All of these mechanisms introduce flavor-violating couplings and new, heavy degrees of freedom. For instance, partial compositeness or warped extra dimensions predict vector-like heavy quarks and colored spin-one resonances with large cross sections, with features which are unfortunately not unique to flavor models. Similar structures appear in alternative models. However, experimental results drive the underlying mass scales into regions which are not accessible by the LHC. This is the reason why theories for quark and lepton flavor physics usually rely on an effective field theory description, neglecting the effects of actually new particles.

We propose search strategies for the dynamic agent of flavor symmetry breaking [6], the flavon, at a future hadron collider. Using a minimal Froggatt-Nielsen setup we only allow for couplings directly related to the generation of the flavor hierarchies. This means that a future discovery can directly probe the underlying mechanism of flavor symmetry breaking. In general, the dimensionless Yukawa couplings do not favor any underlying mass scale; a low flavor breaking scale appears if we link the flavor breaking and the electroweak scales [7] or if dark matter interactions are mediated by flavon exchange [8]. In this paper we deliberately remain agnostic about the ultraviolet completion and discuss the accessible parameter space independent of model building aspect: F $^{*}$

We start by reviewing the most stringent flavor bounds, including projections of current and future experiments testing the quark and lepton sectors. In recent years, significant progress has been made in testing the quark flavor structure at LHC, Belle, and BaBar. Future searches will only slightly increase their sensitivity. On the other hand, searches for lepton flavor effects [9] are entering a golden era with MEG II, Mu3e, DeeMe, COMET, and Mu2e. They should improve existing limits by orders of magnitude. In our setup we see how they will probe parameter regions far beyond the reach of quark flavor physics.

Next, we discuss the discovery reach of the LHC and of a $100 \mathrm{TeV}$ hadron collider [10]. We find that a $100 \mathrm{TeV}$ hadron collider will for the first time allow us to probe a sizeable part of the flavon parameter space, i.e. giving us access to the actual dynamic degrees of freedom in the flavor sector rather than constraining its symmetry structure based on effective field theory. This way, flavon searches add a qualitatively new aspect to the case of a future proton-proton collider, including WIMP dark matter searches [11], Higgs precision measurements [12], searches for new heavy particles [13], and testing mechanisms of baryogenesis [14].

\section{FLAVON MODEL}

In the simplest flavon setup the Higgs and all Standard Model fermions, except for the top, carry charges under a global $U(1)$ or a discrete subgroup. The top Yukawa coupling is then the only allowed renormalizable Yukawa coupling. Introducing a complex scalar field $S$ with flavor charge $a_{S}=1$ we write

$$
\begin{aligned}
-\mathcal{L}_{\text {Yukawa }} & =y_{i j}^{d}\left(\frac{S}{\Lambda}\right)^{n_{i j}^{d}} \bar{Q}_{i} H d_{R_{j}}+y_{i j}^{u}\left(\frac{S}{\Lambda}\right)^{n_{i j}^{u}} \bar{Q}_{i} \widetilde{H} u_{R_{j}} \\
& +y_{i j}^{\ell}\left(\frac{S}{\Lambda}\right)^{n_{i j}^{\ell}} \bar{L}_{i} H \ell_{R_{j}}+y_{i j}^{\nu}\left(\frac{S}{\Lambda}\right)^{n_{i j}^{\nu}} \bar{L}_{i} \widetilde{H} \nu_{R_{j}}+\text { h.c. }
\end{aligned}
$$

The indices $i, j=1,2,3$ link the the fundamental Yukawa couplings $y_{i j}$ with corresponding powers of $S / \Lambda$. The last term assumes the presence of right-handed neutrinos. The field $S$ develops a VEV through a potential

$$
-\mathcal{L}_{\text {potential }}=-\mu_{S}^{2} S^{*} S+\lambda_{S}\left(S^{*} S\right)^{2}+b\left(S^{2}+S^{* 2}\right)+\lambda_{H S}\left(S^{*} S\right)\left(H^{\dagger} H\right)+V(H) .
$$

\footnotetext{
* This includes the obvious application of flavon models to the observed $750 \mathrm{GeV}$ excess which we cannot be bothered to work out (yet).
} 
For now we neglect the portal interaction, $\lambda_{H S}=0$. In its presence, Higgs-flavon mixing [15] and deviations of the Higgs couplings become an alternative strategy to search for the flavon. Under the assumption $\lambda_{H S}=0$ the physical flavon fields is defined by excitations around the VEV,

$$
S(x)=\frac{f+s(x)+i a(x)}{\sqrt{2}} .
$$

The masses of the scalar and pseudo-scalar components are given by

$$
m_{s}=\mu_{S}=\sqrt{\lambda_{S}} f \quad \text { and } \quad m_{a}=\sqrt{2 b} .
$$

This means that the mass of the pseudo-scalar 'pion' of flavor breaking remains a free parameter. It if stays below the flavor scale we can assume the mass hierarchy

$$
m_{a}<m_{s} \approx f<\Lambda .
$$

The pseudoscalar component of the flavon is most likely the first resonance we would encounter in a search for a mechanism behind the flavor structure of the Standard Model. In an abuse of notation, we will therefore refer to it as the pseudoscalar flavon.

The ratio $\epsilon$ of the VEV and the ultraviolet mass scale $\Lambda$ describes the entire flavor structure of the Standard Model,

$$
\epsilon=\frac{f}{\sqrt{2} \Lambda}=\frac{1}{\Lambda} \sqrt{\frac{\mu_{S}^{2}}{2 \lambda_{S}}} \quad \text { with } \quad v<f<\Lambda .
$$

For our numerical analysis we assume that $\epsilon$ is identified with the Cabibbo angle

$$
\epsilon=\left(V_{\mathrm{CKM}}\right)_{12} \approx 0.23 .
$$

The fundamental Yukawa matrices are assumed to be anarchic and of order one

$$
\left|y^{u, d, \ell}\right| \approx\left(\begin{array}{ccc}
1 & 1 & 1 \\
1 & 1 & 1 \\
1 & 1 & 1
\end{array}\right)
$$

Following the Lagrangian given in Eq.(1) the numbers of insertions $n_{i j}$ generate the effective Yukawa couplings

$$
-\mathcal{L}_{\text {Yukawa }}=Y_{i j}^{d} \bar{Q}_{i} H d_{R_{j}}+Y_{i j}^{u} \bar{Q}_{i} \widetilde{H} u_{R_{j}}+Y_{i j}^{\ell} \bar{L}_{i} H \ell_{R_{j}}+Y_{i j}^{\nu} \bar{L}_{i} \widetilde{H} \nu_{R_{j}}+\text { h.c. },
$$

with $Y_{i j}=y_{i j} \epsilon^{n_{i j}}$.

\section{Flavon couplings}

The exponents $n_{i j}$ of the ratio $S / \Lambda$ defined in Eq.(1) can be expressed in terms of the flavor charges of the fermions and Higgs bosons. For the quarks they read

$$
\begin{aligned}
& n_{i j}^{d}=a_{Q_{i}}-a_{d_{j}}-a_{H} \\
& n_{i j}^{u}=a_{Q_{i}}-a_{u_{j}}+a_{H},
\end{aligned}
$$

where $a_{u_{j}}=a_{u, c, t}$ and $a_{d_{j}}=a_{d, s, b}$ denote the flavor charges of the three generations of quark singlets, $a_{Q_{i}}$ are the flavor charges of the three generations of quark doublets, and $a_{H}$ is the flavor charge of the Higgs. To obtain the correct quark masses in our benchmark scenario we set $a_{S}=+1, a_{H}=0$, and

$$
\left(\begin{array}{ccc}
a_{Q_{1}} & a_{Q_{2}} & a_{Q_{3}} \\
a_{u} & a_{c} & a_{t} \\
a_{d} & a_{s} & a_{b}
\end{array}\right)=\left(\begin{array}{ccc}
3 & 2 & 0 \\
-5 & -2 & 0 \\
-4 & -3 & -3
\end{array}\right)
$$


Combined with order-one Yukawa couplings, as spelled out in the Appendix, this gives the quark masses

$$
m_{t} \approx \frac{v}{\sqrt{2}} \quad \frac{m_{b}}{m_{t}} \approx \epsilon^{3} \quad \frac{m_{c}}{m_{t}} \approx \epsilon^{4} \quad \frac{m_{s}}{m_{t}} \approx \epsilon^{5} \quad \frac{m_{d}}{m_{t}} \approx \epsilon^{7} \quad \frac{m_{u}}{m_{t}} \approx \epsilon^{8},
$$

and the CKM matrix becomes

$$
V_{\mathrm{CKM}} \approx\left(\begin{array}{ccc}
1 & \epsilon & \epsilon^{3} \\
\epsilon & 1 & \epsilon^{2} \\
\epsilon^{3} & \epsilon^{2} & 1
\end{array}\right),
$$

The flavon couplings to fermions in the mass eigenbasis are linked to the Yukawa couplings,

$$
g_{a f_{i L} f_{j R}}^{u} \equiv g_{a i j}^{u}=\frac{1}{f}\left(\begin{array}{ccc}
8 m_{u} & \epsilon m_{c} & \epsilon^{3} m_{t} \\
\epsilon^{3} m_{c} & 4 m_{c} & \epsilon^{2} m_{t} \\
\epsilon^{5} m_{t} & \epsilon^{2} m_{t} & 0
\end{array}\right) \quad g_{a i j}^{d}=\frac{1}{f}\left(\begin{array}{ccc}
7 m_{d} & \epsilon m_{s} & \epsilon^{3} m_{b} \\
\epsilon m_{s} & 5 m_{s} & \epsilon^{2} m_{b} \\
\epsilon m_{b} & \epsilon^{2} m_{b} & 3 m_{b}
\end{array}\right) .
$$

where in the off-diagonal terms we neglect order-one factors. The fact that the flavon does not couple to top quarks reflects our assumption that the corresponding term in the Lagrangian starts at $\epsilon^{0}$, i.e. without any suppression $f / \Lambda$.

In the lepton sector the analogous exponents in Eq.(1) are given by

$$
\begin{aligned}
& n_{i j}^{\ell}=a_{L_{i}}-a_{\ell_{j}}-a_{H} \\
& n_{i j}^{\nu}=a_{L_{i}}-a_{\nu_{j}}+a_{H},
\end{aligned}
$$

in terms of the ten flavor charges. As in the quark sector, we choose the charges to reproduce the lepton masses and mixing patterns,

$$
\left(\begin{array}{ccc}
a_{L_{1}} & a_{L_{2}} & a_{L_{3}} \\
a_{\nu_{e}} & a_{\nu_{\mu}} & a_{\nu_{\tau}} \\
a_{e} & a_{\mu} & a_{\tau}
\end{array}\right)=\left(\begin{array}{ccc}
1 & 0 & 0 \\
-24 & -21 & -20 \\
-8 & -5 & -3
\end{array}\right)
$$

The neutrino charges can be smaller if a Majorana mass term exists. One attractive way to implement it is to assume a flavor charge of $\nu_{R}=1 / 2$, such that

$$
\mathcal{L}_{\text {Majorana }}=M_{\nu} \nu_{R} \nu_{R},
$$

with $M_{\nu}=f$. This gives us the lepton mass ratios

$$
\frac{m_{\tau}}{m_{t}} \approx \epsilon^{3} \quad \frac{m_{\mu}}{m_{t}} \approx \epsilon^{5} \quad \frac{m_{e}}{m_{t}} \approx \epsilon^{9} \quad \frac{m_{\nu_{1}}}{m_{t}} \approx \epsilon^{25} \quad \frac{m_{\nu_{2}}}{m_{t}} \approx \epsilon^{21} \quad \frac{m_{\nu_{3}}}{m_{t}} \approx \epsilon^{20},
$$

and the leptonic mixing matrix

$$
U_{\mathrm{PMNS}} \approx\left(\begin{array}{lll}
1 & \epsilon & \epsilon \\
\epsilon & 1 & 1 \\
\epsilon & 1 & 1
\end{array}\right)
$$

Again, the flavon couplings are related to the Yukawa couplings, modulo order-one corrections in the offdiagonal terms of

$$
g_{a f_{i L} f_{j R}}^{\ell} \equiv g_{a i j}^{\ell}=\frac{1}{f}\left(\begin{array}{ccc}
9 m_{e} & \epsilon m_{\mu} & \epsilon m_{\tau} \\
\epsilon^{3} m_{\mu}^{3} & 5 m_{\mu} & \epsilon^{2} m_{\tau} \\
\epsilon^{5} m_{\tau} & \epsilon^{2} m_{\tau} & 3 m_{\tau}
\end{array}\right)
$$

In all cases the corresponding scalar couplings to fermions, except for the top Yukawa, can be read off Eq. 14 and Eq. 20). Following the field definition in Eq.(3) we use the notation

$$
g_{i j} \equiv g_{s f_{i L} f_{j R}}=i g_{a f_{i L} f_{j R}}
$$

to leading order and for all fermions except for $i=j=t$. 

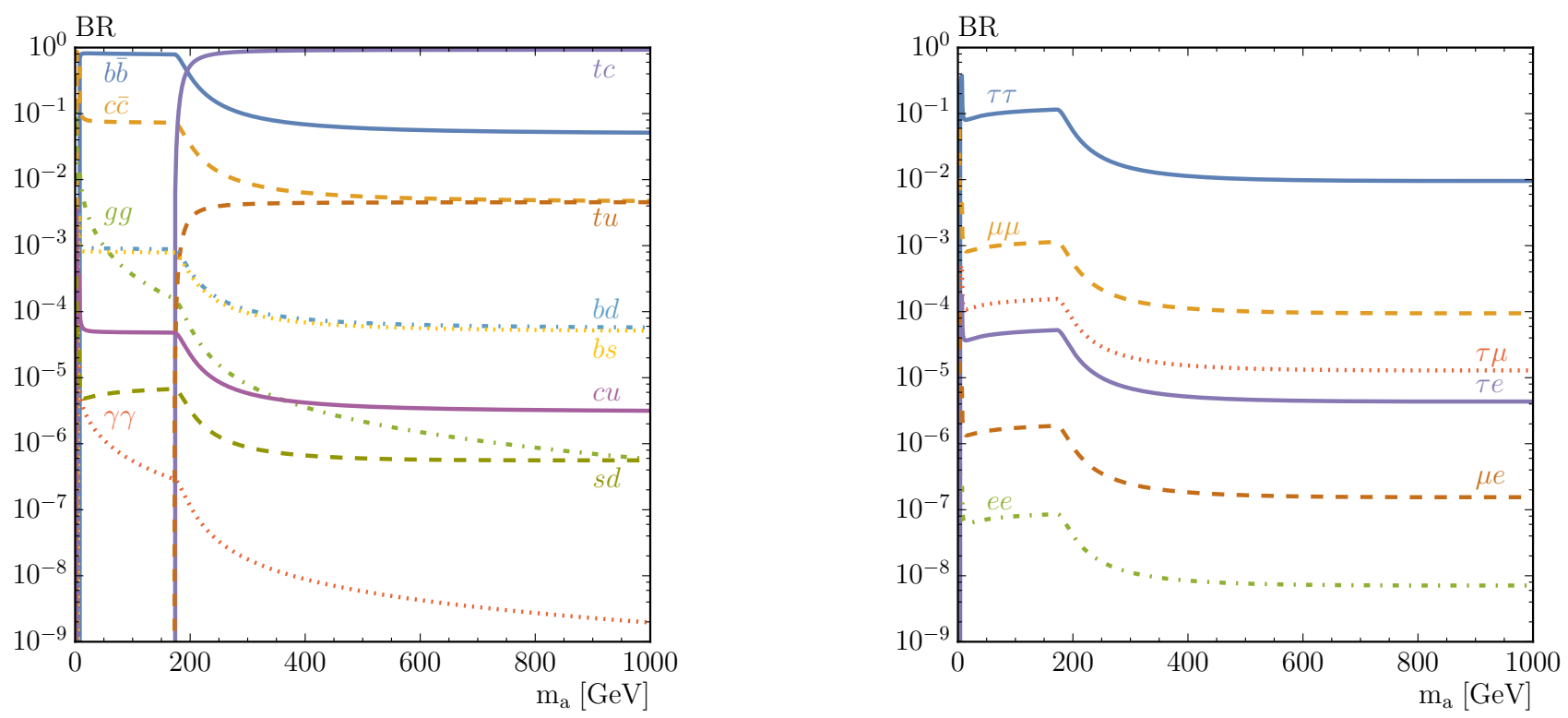

Figure 1. Flavon branching ratios for decays to quarks (left) and leptons (right).

Flavon and top decays

In terms of these flavon couplings to fermions we can compute the flavon branching ratios, which will guide us to possible signatures at colliders. Obviously, flavon decays to a pair of fermions occur at tree level, but unlike for example a Higgs boson the decays do not have to be flavor-diagonal. The general form of the corresponding partial width is

$$
\begin{aligned}
\frac{\Gamma\left(a \rightarrow f_{i} \bar{f}_{j}\right)}{m_{a}}= & \frac{N_{c}}{16 \pi}\left[\frac{\left(m_{a}^{2}-\left(m_{i}+m_{j}\right)^{2}\right)\left(m_{a}^{2}-\left(m_{i}-m_{j}\right)^{2}\right)}{m_{a}^{4}}\right]^{1 / 2} \\
& {\left[\left(\left|g_{i j}\right|^{2}+\left|g_{j i}\right|^{2}\right)\left(1-\frac{m_{i}^{2}+m_{j}^{2}}{m_{a}^{2}}\right)-2\left(g_{i j} g_{j i}+g_{i j}^{*} g_{j i}^{*}\right) \frac{m_{i} m_{j}}{m_{a}^{2}}\right] . }
\end{aligned}
$$

In addition, we can compute the loop-induced partial widths to gluons or photons in complete analogy to the Higgs. The numerical results for our parameter choice $\epsilon=0.23$ are given in Fig. 1. As long as $m_{a}<m_{t}$ the main decay channels are similar to the Higgs, with $a \rightarrow b \bar{b}$ dominating over $a \rightarrow \tau \tau$ due to the larger Yukawa coupling and the color factor $N_{c}$. Above the top threshold almost all pseudoscalar flavons decay to

$$
a \rightarrow t j+\bar{t} j \quad \text { with } \quad \frac{\Gamma(a \rightarrow t \bar{u})}{\Gamma(a \rightarrow t \bar{c})} \approx \epsilon^{2} \approx \frac{1}{20} .
$$

The one obvious question for colliders searches will be if the charm in the final state could be tagged to improve a top+jet resonance signal. In our analysis we do not employ charm tagging and instead leave it as an obvious experimentally driven improvement. Following the construction of the Lagrangian without a suppression $f / \Lambda$ in the top Yukawa, the diagonal decay $a \rightarrow t \bar{t}$ does not occur at tree level.

We can turn around the above discussion, which lead to the dominant flavor-violating flavon decay shown in Eq. (23): according to Eq.(14) the couplings $g_{t c} \sim g_{c t}$ scale like $\epsilon^{2} m_{t} / f \approx m_{t} /(20 f)$. In the limit $m_{c} \ll$ $m_{a}<m_{t}$, the corresponding flavor-changing top decay width is given by

$$
\frac{\Gamma(t \rightarrow c a)}{m_{t}}=\frac{1}{32 \pi}\left(\left|g_{c t}\right|^{2}+\left|g_{t c}\right|^{2}\right)\left(1-\frac{m_{a}^{2}}{m_{t}^{2}}\right)^{2} .
$$

Anomalous top decays into Higgs and charm final states have been searched for at LHC and will be discussed in detail in Sec. V. 


\section{QUARK FLAVOR CONSTRAINTS}

To date, the most constraining measurements on our flavon model arise from quark flavor physics. As usual, loop-induced meson mixing and rare decays have the largest impact on our model, parameterized by the flavon mass $m_{a}$, the $\operatorname{VEV~} f$, and the quartic coupling $\lambda_{S}$.

\section{Neutral meson mixing}

Because flavon models with the coupling structure given in Eq.(14) lead to flavor-changing neutral currents, strong limits are expected from meson anti-meson mixing. The effective Hamiltonian describing $\Delta F=2$ interactions reads

$$
\begin{aligned}
\mathcal{H}_{\mathrm{NP}}^{\Delta F=2} & =C_{1}^{i j}\left(\bar{q}_{L}^{i} \gamma_{\mu} q_{L}^{j}\right)^{2}+\widetilde{C}_{1}^{i j}\left(\bar{q}_{R}^{i} \gamma_{\mu} q_{R}^{j}\right)^{2}+C_{2}^{i j}\left(\bar{q}_{R}^{i} q_{L}^{j}\right)^{2}+\widetilde{C}_{2}^{i j}\left(\bar{q}_{L}^{i} q_{R}^{j}\right)^{2} \\
& +C_{4}^{i j}\left(\bar{q}_{R}^{i} q_{L}^{j}\right)\left(\bar{q}_{L}^{i} q_{R}^{j}\right)+C_{5}^{i j}\left(\bar{q}_{L}^{i} \gamma_{\mu} q_{L}^{j}\right)\left(\bar{q}_{R}^{i} \gamma^{\mu} q_{R}^{j}\right)+\text { h.c. }
\end{aligned}
$$

At tree-level, flavon exchange generates the Wilson coefficients [16, 17]

$$
\begin{aligned}
C_{2}^{i j} & =-\left(g_{j i}^{*}\right)^{2}\left(\frac{1}{m_{s}^{2}}-\frac{1}{m_{a}^{2}}\right) \\
\tilde{C}_{2}^{i j} & =-g_{i j}^{2}\left(\frac{1}{m_{s}^{2}}-\frac{1}{m_{a}^{2}}\right) \\
C_{4}^{i j} & =-\frac{g_{i j} g_{j i}}{2}\left(\frac{1}{m_{s}^{2}}+\frac{1}{m_{a}^{2}}\right) .
\end{aligned}
$$

For $m_{a}=m_{s}$ the two contributions to $C_{2}$ and $\widetilde{C}_{2}$ cancel, while there is a constructive interference in $C_{4}$. Given that the masses in Eq.(4) are set by independent scales, such a cancellation would be accidental. Depending on the meson system, there can be sizable enhancement from RG running and matrix elements. We implement RG running according to Refs. [18, 19] with the matrix elements given in Refs. [20], matching the scalar and
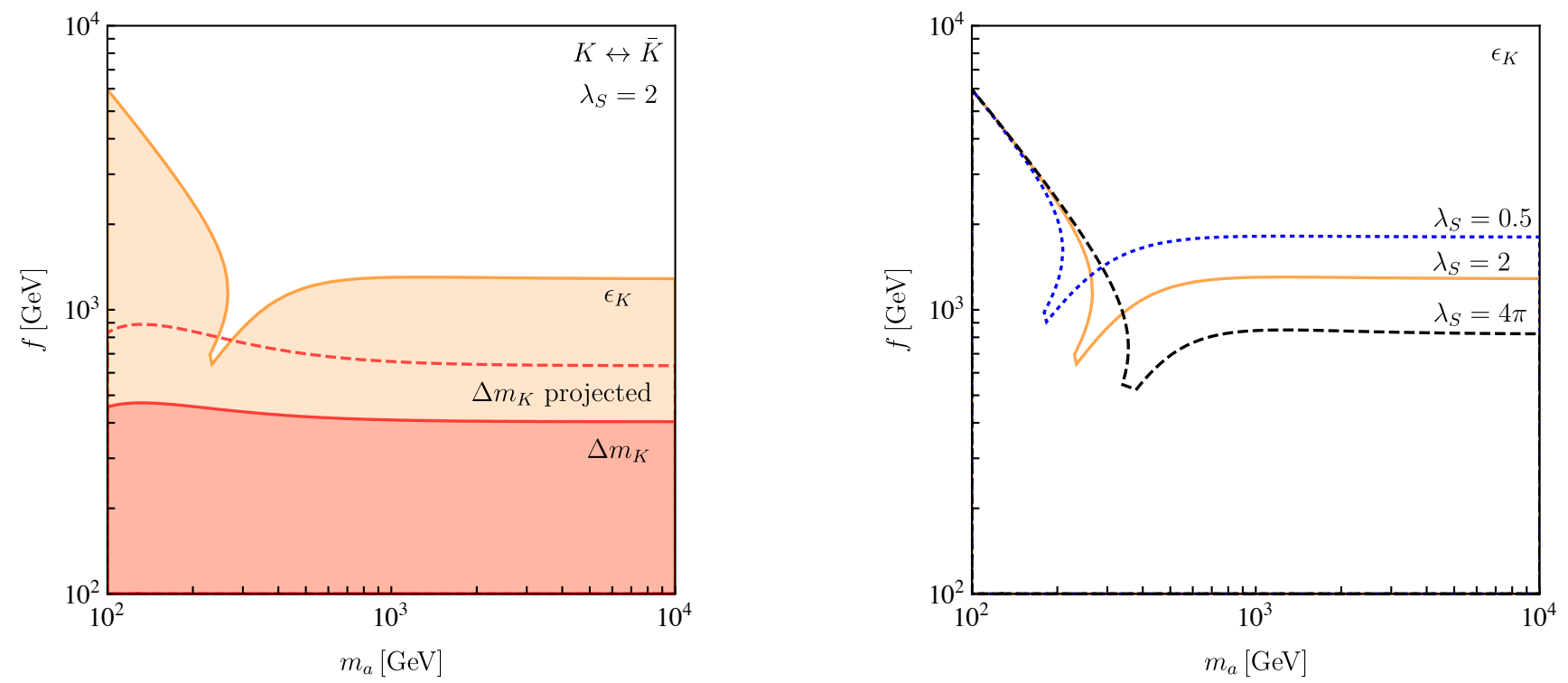

Figure 2. Left: regions in the $m_{a}-f$ plane excluded by flavon contributions to $\epsilon_{K}$ (orange) and $\Delta m_{K}$ (red) for our benchmark point and $\lambda_{S}=2$. The dashed red contour corresponds to the excluded region based on projected improvements in $\Delta m_{K}$. Right: constraint from $\epsilon_{K}$ for $\lambda_{S}=0.5$ (dotted blue), $\lambda_{S}=2$ (orange) and $\lambda_{S}=4 \pi$ (dashed black). 

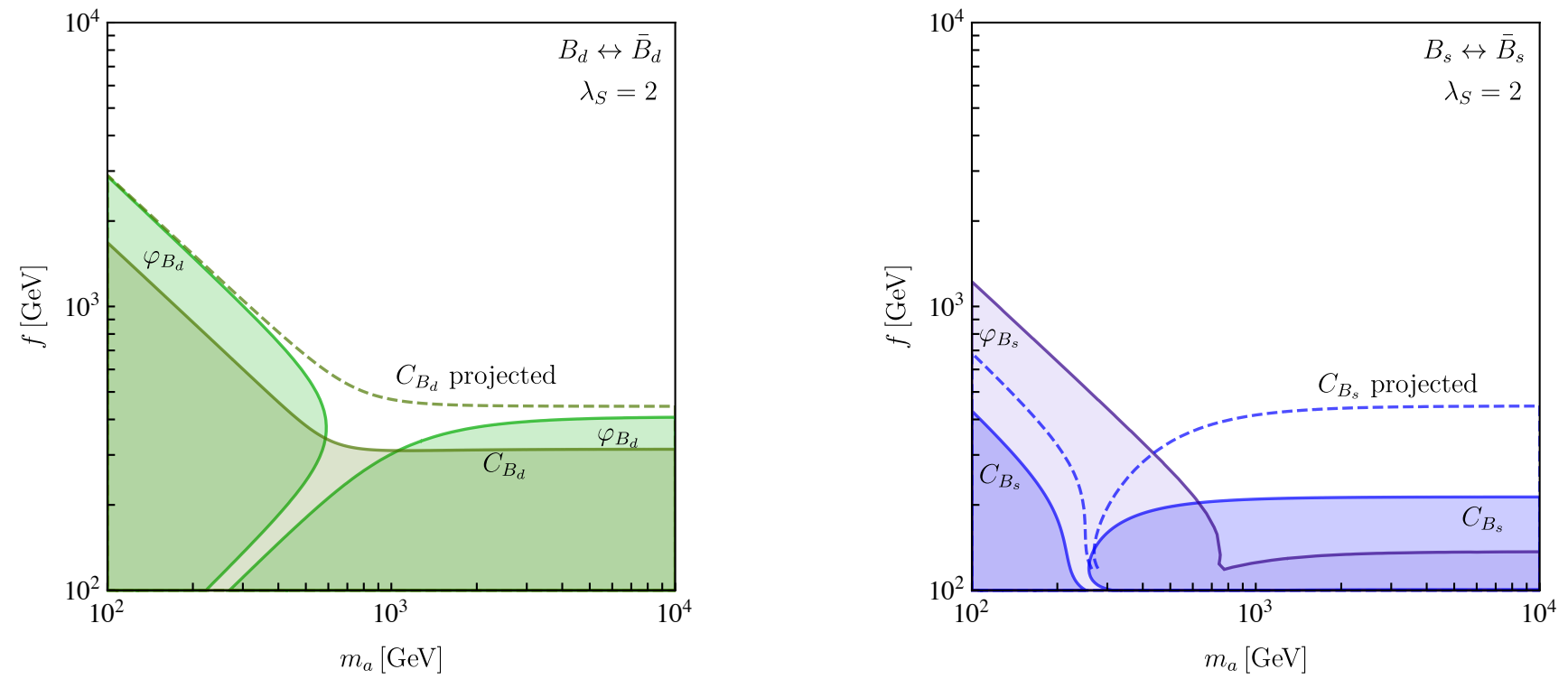

Figure 3. Left: regions in the $m_{a}-f$ plane excluded by flavon contributions to $C_{B_{d}}$ (light green) and $\varphi_{B_{d}}$ (green) for our benchmark point and $\lambda_{S}=2$. Right: constraints from flavon contributions to $C_{B_{s}}$ (blue) and $\varphi_{B_{s}}$ (light purple). The dashed contours correspond to the excluded regions based on projected improvements in $C_{B_{d}}$ and $C_{B_{s}}$.

pseudoscalar flavon contributions at $\mu=m_{s}$ and $\mu=m_{a}$, respectively. Fits based on projections of future experimental improvements on meson mixing observables from LHCb and Belle II, as well as projected lattice improvements are collected in Ref. [21].

We start with $95 \%$ CL limits from $K-\bar{K}$ mixing [18]

$$
\begin{aligned}
C_{\epsilon_{K}} & =\frac{\operatorname{Im}\left\langle K^{0}\left|\mathcal{H}^{\Delta F=2}\right| \bar{K}^{0}\right\rangle}{\operatorname{Im}\left\langle K^{0}\left|\mathcal{H}_{\mathrm{SM}}^{\Delta F=2}\right| \bar{K}^{0}\right\rangle}=1.05_{-0.28}^{+0.36} \\
C_{\Delta m_{K}}= & \frac{\operatorname{Re}\left\langle K^{0}\left|\mathcal{H}^{\Delta F=2}\right| \bar{K}^{0}\right\rangle}{\operatorname{Re}\left\langle K^{0}\left|\mathcal{H}_{\mathrm{SM}}^{\Delta F=2}\right| \bar{K}^{0}\right\rangle}=0.93_{-0.42}^{+1.14} .
\end{aligned}
$$

where $\mathcal{H}^{\Delta F=2}$ includes the SM and flavon contributions, while $\mathcal{H}_{\mathrm{SM}}^{\Delta F=2}$ parameterizes the SM contribution. In the left panel of Fig. 2 we show the region excluded by contributions from scalar and pseudoscalar flavon exchange to $C_{\epsilon_{K}}$ and $C_{\Delta m_{K}}$. The dip feature is due to the accidental cancellation in $C_{2}^{s d}$ and $\tilde{C}_{2}^{s d}$, as shown in Eq. (26). It is a universal feature in $K-\bar{K}$ mixing, unless the contribution to $C_{4}^{s d}$ completely dominates. For our benchmark point, the dip in $C_{\Delta m_{K}}$ is below $m_{a}=100 \mathrm{GeV}$, not visible in the plot. The position also depends on the scalar quartic $\lambda_{S}$, which also determines the excluded value of $f$ for large $m_{a}$. The dashed red contour corresponds to the excluded region based on projected improvements in $\Delta m_{K}$, under the optimistic assumptions presented in Ref. [21]. The right panel of Figure 2 shows the variation in the $C_{\epsilon_{K}}$ exclusion contour for $\lambda_{S}=0.5,2,4 \pi$.

For the two versions of $B-\bar{B}$ mixing we define

$$
C_{B_{q}} e^{2 i \varphi_{B_{q}}}=\frac{\left\langle B_{q}\left|\mathcal{H}^{\Delta F=2}\right| \bar{B}_{q}\right\rangle}{\left\langle B_{q}\left|\mathcal{H}_{\mathrm{SM}}^{\Delta F=2}\right| \bar{B}_{q}\right\rangle},
$$

with the $95 \%$ CL limits 18

$$
\begin{array}{ll}
C_{B_{d}}=1.07_{-0.31}^{+0.36} & \varphi_{B_{d}}=-2.0_{-6.0}^{+6.4} \\
C_{B_{s}}=1.052_{-0.152}^{+0.178} & \varphi_{B_{s}}=0.72_{-2.28}^{+3.98} .
\end{array}
$$




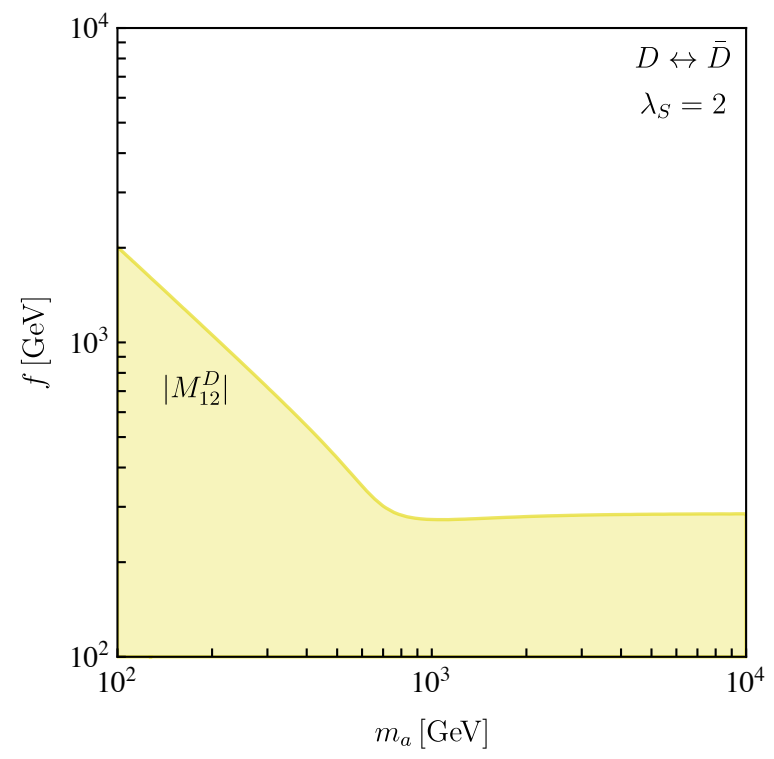

Figure 4. Regions in the $m_{a}-f$ plane excluded by flavon contributions to $\left|M_{12}^{D}\right|$ (shaded yellow) for our benchmark point and $\lambda_{S}=2$.

Figure 3 shows the excluded regions in the $f-m_{a}$ plane for our benchmark point. The optimistic projected improvements in $C_{B_{d}}$ and $C_{B_{s}}$ follow Ref. [21].

Finally, since the SM contribution to $D-\bar{D}$ mixing is plagued with very large hadronic uncertainties, we define and only demand that the flavon contributions do not exceed the $2 \sigma$ constraint [22]

$$
\left|M_{12}^{D}\right|=\mid\left\langle D\left|\mathcal{H}^{\Delta F=2}\right| \bar{D}\right\rangle<7.7 \mathrm{ps}^{-1} .
$$

The results for our benchmark point are shown in Fig. 4. In principle, the sizable flavon coupling $g_{t c, c t}$ could result in sizable loop contributions from one-loop box diagram. Altogether, we find a relative suppression of the kind $m_{t}^{2} \epsilon^{2} /\left(4 \pi^{2} f^{2}\right)$ with respect to the tree level diagram, which renders the loop contributions completely negligible for the parameter space of interest.

\section{Leptonic meson decays}

Flavon-mediated decays of neutral mesons into charged leptons can be described by the effective Hamiltonian

$$
\mathcal{H}_{\mathrm{eff}}=-\frac{G_{F}^{2} m_{W}^{2}}{\pi^{2}}\left(C_{S}^{i j}\left(\bar{q}_{i} P_{L} q_{j}\right) \bar{\ell} \ell+\tilde{C}_{S}^{i j}\left(\bar{q}_{i} P_{R} q_{j}\right) \bar{\ell} \ell+C_{P}^{i j}\left(\bar{q}_{i} P_{L} q_{j}\right) \bar{\ell} \gamma_{5} \ell+\tilde{C}_{P}^{i j}\left(\bar{q}_{i} P_{R} q_{j}\right) \bar{\ell} \gamma_{5} \ell\right)+\text { h.c. }
$$

The branching ratio for the meson decay of a neutral meson is given by

$$
\begin{aligned}
\operatorname{BR}\left(M \rightarrow \ell^{+} \ell^{-}\right)= & \frac{G_{F}^{4} m_{W}^{4}}{8 \pi^{5}} \beta m_{M} f_{M}^{2} m_{\ell}^{2} \tau_{M} \\
& {\left[\left|\frac{m_{M}^{2}\left(C_{P}^{i j}-\tilde{C}_{P}^{i j}\right)}{2 m_{\ell}\left(m_{i}+m_{j}\right)}-C_{A}^{\mathrm{SM}}\right|^{2}+\left|\frac{m_{M}^{2}\left(C_{S}^{i j}-\tilde{C}_{S}^{i j}\right)}{2 m_{\ell}\left(m_{i}+m_{j}\right)}\right|^{2} \beta^{2}\right], }
\end{aligned}
$$



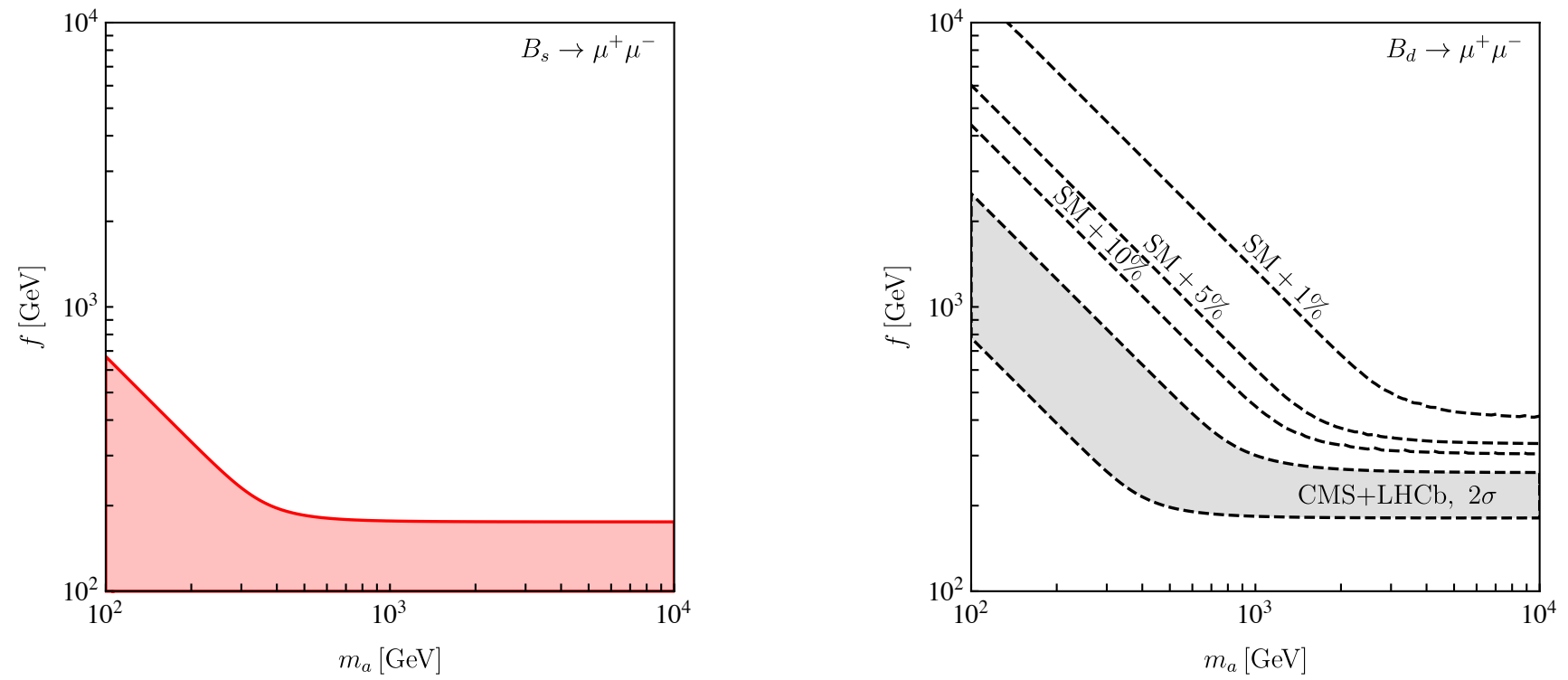

Figure 5. Left: regions in the $m_{a}-f$ plane excluded by flavon contributions to the decay $B_{s} \rightarrow \mu^{+} \mu^{-}$. Right: parameter space where the branching ratio for $B_{d} \rightarrow \mu^{+} \mu^{-}$stays within the $2 \sigma$ confidence interval (shaded gray), as well as contours of $1 \%, 5 \%$ and $10 \%$ enhancement with respect to the SM prediction.

where $\beta(x)=\sqrt{1-4 x^{2}}$ with $x=m_{\ell} / m_{M}$. As for meson mixing, we only need to consider tree-level flavon contributions to the corresponding Wilson coefficients,

$$
\begin{aligned}
C_{S}^{i j} & =\frac{\pi^{2}}{2 G_{F}^{2} m_{W}^{2}} \frac{2 g_{\ell \ell} g_{j i}}{m_{s}^{2}} & \tilde{C}_{S}^{i j} & =\frac{\pi^{2}}{2 G_{F}^{2} m_{W}^{2}} \frac{2 g_{\ell \ell} g_{i j}}{m_{s}^{2}} \\
C_{P}^{i j} & =\frac{\pi^{2}}{2 G_{F}^{2} m_{W}^{2}} \frac{2 g_{\ell \ell} g_{j i}}{m_{a}^{2}} & \tilde{C}_{P}^{i j} & =\frac{\pi^{2}}{2 G_{F}^{2} m_{W}^{2}} \frac{2 g_{\ell \ell} g_{i j}}{m_{a}^{2}} .
\end{aligned}
$$

Since the scalar contributions do not interfere with the SM contribution, the resulting constraints are almost independent from the scalar mass. In addition, they are insensitive to the value of the quartic coupling $\lambda_{S}$. In contrast, the SM contribution is generated at one loop, and for the $B_{s}$-system is to a very good approximation given by

$$
C_{\mathrm{SM}}=-V_{t b}^{*} V_{t s} Y\left(\frac{m_{t}^{2}}{m_{W}^{2}}\right)
$$

with

$$
Y(x)=\eta_{\mathrm{QCD}} \frac{x}{8}\left[\frac{4-x}{1-x}+\frac{3 x}{(1-x)^{2}} \log x\right],
$$

where $\eta_{\mathrm{QCD}}=1.0113$ parametrizes higher order corrections [23]. Due to the sizable width difference of the $B_{s}$-meson system, the theoretical prediction has to be rescaled by $\left(1-y_{s}\right)^{-1}$, where $y_{s}=0.088 \pm 0.014$ [24], before being compared with the experimental result [25].

$$
\mathrm{BR}\left(B_{s} \rightarrow \mu^{+} \mu^{-}\right)=2.8_{-0.6}^{+0.7} \cdot 10^{-9}
$$

The corresponding limits on our flavon benchmark point are shown in the left panel of Fig. 5. In the case of the $B_{d}$-system, the corresponding correction is negligible and the SM prediction follows from a straightforward replacement of indices in Eq. 34]. The recent combination of CMS [26] and LHCb [27] measurements yields [25]

$$
\mathrm{BR}\left(B_{d} \rightarrow \mu^{+} \mu^{-}\right)=(3.6 \pm 1.6) \cdot 10^{-10} .
$$


For this channel we require our flavon contributions to stay within the $2 \sigma$ interval, namely $\operatorname{BR}\left(B_{d} \rightarrow \mu^{+} \mu^{-}\right)=$ $[1.4,7.4] \cdot 10^{-10}$. In the right panel of Fig. 5 we show where the flavon contributions agree with measured value of $\operatorname{BR}\left(B_{d} \rightarrow \mu^{+} \mu^{-}\right)$, as well as $1 \%, 5 \%$ and $10 \%$ enhancements with respect to the SM prediction. While an explanation of the $2 \sigma$ deviation is in tension with constraints from neutral meson mixing, flavon exchange can lead to sizable enhancements.

In addition to these bottom-mesons we can also derive constraints from $D \rightarrow \mu^{+} \mu^{-}$decays, which turn out considerably weaker. Finally, flavon limits from $K_{L} \rightarrow \mu^{+} \mu^{-}$decays exclude a region in parameter space very similar to the one ruled out by $B_{s} \rightarrow \mu^{+} \mu^{-}$.

\section{FUTURE LEPTON FLAVOR MEASUREMENTS}

While currently the experimental results from quark flavor physics are most constraining for our flavon models, we discuss a set of upcoming experiments in lepton flavor physics which will dramatically improve in the coming years. We can use the same benchmark point for these lepton flavor experiments as for collider searches, because the lepton and quark sectors of our flavon models can be adapted independently.

$$
\text { Decay } \mu \rightarrow e \gamma
$$

Radiative leptonic decays are mediated by dipole operators

$$
\mathcal{L}_{\text {eff }}=m_{\ell^{\prime}} C_{T}^{L} \bar{\ell} \sigma^{\rho \lambda} P_{L} \ell^{\prime} F_{\rho \lambda}+m_{\ell^{\prime}} C_{T}^{R} \bar{\ell} \sigma^{\rho \lambda} P_{R} \ell^{\prime} F_{\rho \lambda} .
$$

giving a branching ratio

$$
\operatorname{BR}\left(\ell^{\prime} \rightarrow \ell \gamma\right)=\frac{m_{\ell^{\prime}}^{5}}{4 \pi \Gamma_{\ell^{\prime}}}\left(\left|C_{T}^{L}\right|^{2}+\left|C_{T}^{R}\right|^{2}\right)
$$

The relevant one-loop diagram for the flavon contribution, shown in Fig. 6, gives the Wilson coefficients

$$
\begin{aligned}
C_{T}^{L}=\left(C_{T}^{R}\right)^{*}=\frac{g}{32 \pi^{2}} \sum_{k=e, \mu, \tau} & \left\{\frac{1}{6}\left(g_{\ell k}^{*} g_{\ell^{\prime} k}+\frac{m_{\ell}}{m_{k}} g_{k \ell}^{*} g_{k \ell^{\prime}}\right)\left(\frac{1}{m_{s}^{2}}-\frac{1}{m_{a}^{2}}\right)\right. \\
& \left.-g_{\ell k} g_{k \ell^{\prime}} \frac{m_{k}}{m_{\ell^{\prime}}}\left[\frac{1}{m_{s}^{2}}\left(\frac{3}{2}+\log \frac{m_{\ell^{\prime}}^{2}}{m_{s}^{2}}\right)-\frac{1}{m_{a}^{2}}\left(\frac{3}{2}+\log \frac{m_{\ell^{\prime}}^{2}}{m_{a}^{2}}\right)\right]\right\} .
\end{aligned}
$$

In particular for $\mu \rightarrow e \gamma$ the chirally enhanced second term in Eq.40 leads to sizable contributions. Current experimental bounds are [28, 29]

$$
\mathrm{BR}(\mu \rightarrow e \gamma)<5.7 \cdot 10^{-13} \quad \text { and } \quad \mathrm{BR}(\tau \rightarrow \mu \gamma)<4.5 \cdot 10^{-8},
$$

while the upgraded MEG II experiment has a projected sensitivity of [30]

$$
\mathrm{BR}(\mu \rightarrow e \gamma)=6 \cdot 10^{-14} \text {. }
$$

In Fig. 7 we show the different constraints on our flavon model.
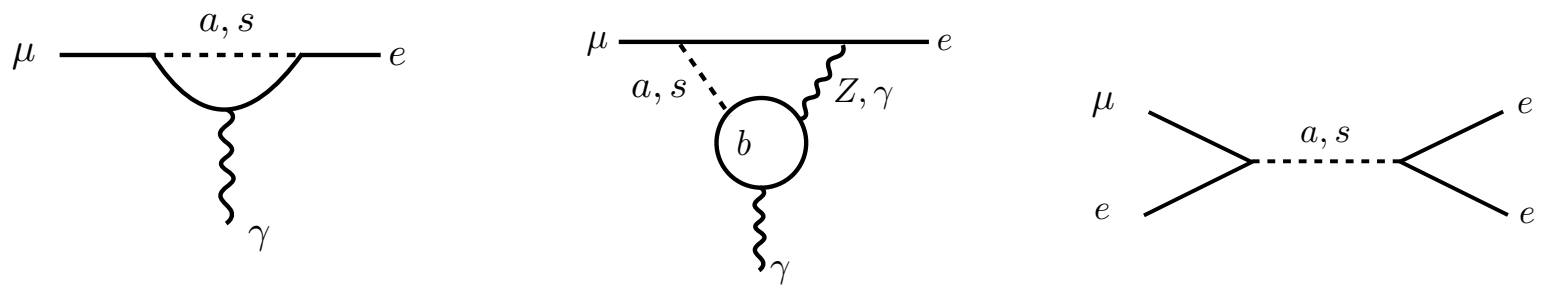

Figure 6. Feynman diagrams showing flavon contributions to $\mu \rightarrow e \gamma$ at one-loop level and two-loop level, as well as flavon contributions to $\mu \rightarrow 3 e$. 


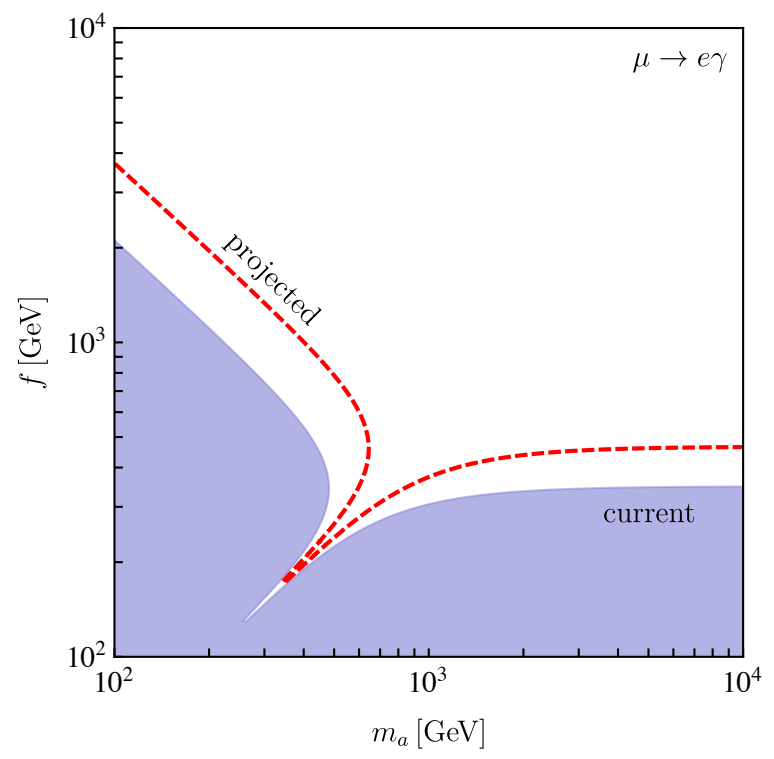

Figure 7. Regions in the $m_{a}-f$ plane excluded by flavon contributions to $\mu \rightarrow e \gamma$. The corresponding bounds from $\tau \rightarrow \mu \gamma$ and $\tau \rightarrow e \gamma$ are not visible for the plotted parameter range.

\section{Conversion $\mu \rightarrow e$}

In addition to the dipole operators shown in Eq.(38), the following effective operators contribute to $N \mu \rightarrow$ $\mathrm{Ne}$ conversion

$$
\mathcal{L}_{\mathrm{eff}}=C_{q q}^{V L} \bar{e} \gamma^{\nu} P_{L} \mu \bar{q} \gamma_{\nu} q+m_{\mu} m_{q} C_{q q}^{S L} \bar{e} P_{R} \mu \bar{q} q+m_{\mu} \alpha_{s} C_{g g}^{L} \bar{e} P_{R} \mu G_{\rho \nu} G^{\rho \nu}+(R \leftrightarrow L),
$$

Before we include the nuclear effects to compute the actual conversion rate, we derive the Wilson coefficients induced by flavon exchange. The relevant diagram is shown on the left of Fig. 8 and gives us

$$
\begin{aligned}
C_{q q}^{S L} & =\left(\frac{1}{m_{s}^{2}}+\frac{1}{m_{a}^{2}}\right) g_{\mu e}^{*} \operatorname{Re}\left(g_{q q}\right), \\
C_{q q}^{S R} & =\left(\frac{1}{m_{s}^{2}}-\frac{1}{m_{a}^{2}}\right) g_{e \mu} \operatorname{Re}\left(g_{q q}\right) .
\end{aligned}
$$

Contributions to $C_{g g}^{L, R}$ arise only from integrating out the non-dynamical heavy quarks and we absorb them in $\tilde{C}_{p}^{S L}$ and $\tilde{C}_{n}^{S L}$. The relevant diagram is shown on the right hand side of Fig. 8 . We further confirm that contributions from vector operators are smaller than all scalar Wilson coefficients and can be neglected [31]. Barr-Zee-type diagrams, as shown in Fig. 6, which generate the dominant contributions to both $\mu \rightarrow e \gamma$ and $\mu \rightarrow e$ conversion for lepton flavor violating Higgs couplings are small due to the absence of couplings to the top quark.
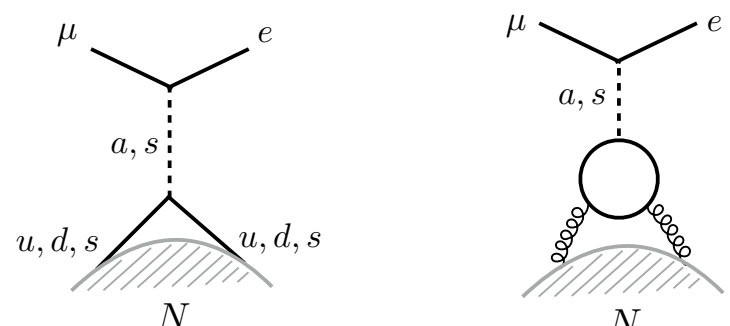

$N$

Figure 8. Diagrams showing flavon contributions to $\mu \rightarrow e$ conversion in nuclei at tree level and one-loop level. 


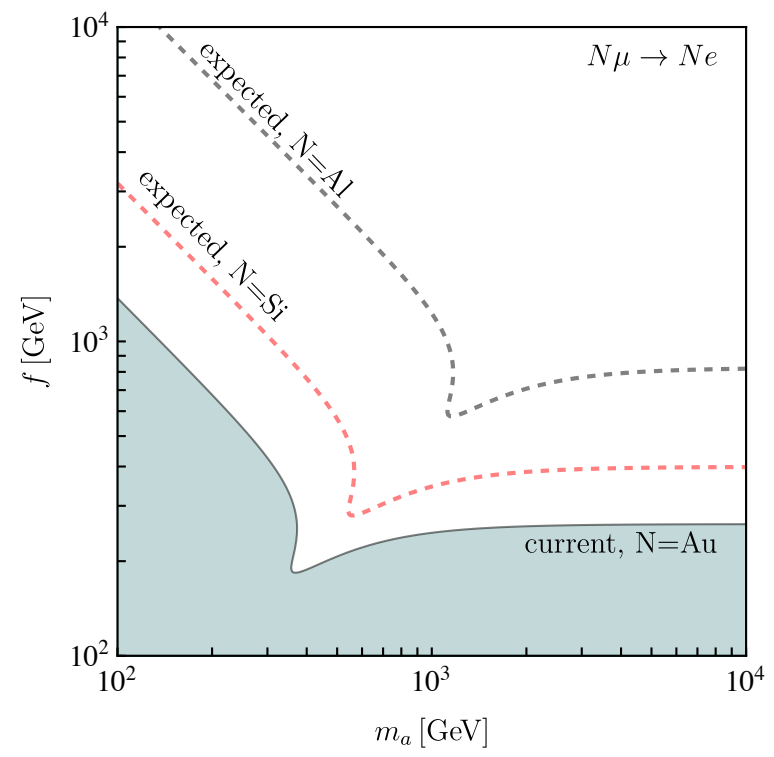

Figure 9. Regions in the $m_{a}-f$ plane excluded by flavon contributions to the conversion $N \mu \rightarrow N e$.

Next, we need to account for the effects of quarks inside the nucleons. We define the nucleon-level Wilson coefficients

$$
\tilde{C}_{p}^{V L}=\sum_{q=u, d} C_{q q}^{V L} f_{V_{q}}^{p} \quad \text { and } \quad \tilde{C}_{p}^{S L}=\sum_{q=u, d, s} C_{q q}^{S L} f_{q}^{p}-\sum_{Q=c, b, t} C_{Q Q}^{S L} f_{\text {heavy }}^{p},
$$

in which $f_{V_{q}}^{p}, f_{q}^{p}$, and $f_{\text {heavy }}^{p}=2 / 27\left(1-f_{u}^{p}-f_{d}^{p}-f_{s}^{p}\right)$ account for the quark content of the proton [32]. Analogous expressions hold for the neutron. We use the numbers given in Refs. 33, 34, based on the lattice average from Ref. [35,

$$
\begin{array}{ll}
f_{u}^{p}=0.0191 & f_{u}^{n}=0.0171, \\
f_{d}^{p}=0.0363 & f_{d}^{n}=0.0404, \\
f_{s}^{p}=f_{s}^{n}=0.043 . &
\end{array}
$$

Using the $\sigma$-term derived from $S U(3)_{C}$ relations does not change the results qualitatively. Finally, we can compute the conversion rate including effects from the nucleus' structure,

$$
\Gamma_{N \mu \rightarrow N e}=\frac{m_{\mu}^{5}}{4}\left|C_{T}^{L} D+4\left[m_{\mu} m_{p} \tilde{C}_{p}^{S L}+\tilde{C}_{p}^{V L} V^{p}+(p \rightarrow n)\right]\right|^{2},
$$

with $p$ and $n$ denoting the proton and neutron, respectively. The coefficients $D, S^{p, n}$ and $V^{p, n}$ are dimensionless functions of the overlap integrals of the initial state muon and the final-state electron wave-functions with the target nucleus. We use the numerical values [36]

\begin{tabular}{l|cccccc}
\hline Target & $D$ & $S^{p}$ & $S^{n}$ & $V^{p}$ & $V^{n}$ & $\Gamma_{\text {capt }}\left[10^{-6} \mathrm{~s}\right]$ \\
\hline $\mathrm{Au}$ & 0.189 & 0.0614 & 0.0918 & 0.0974 & 0.146 & 13.06 \\
$\mathrm{Al}$ & 0.0362 & 0.0155 & 0.0167 & 0.0161 & 0.0173 & 0.705 \\
$\mathrm{Si}$ & 0.0419 & 0.0179 & 0.0179 & 0.0187 & 0.0187 & 0.871 \\
\hline
\end{tabular}

with $\Gamma_{\text {capt }}$ denoting the muon capture rate.

Currently, the strongest experimental bound on $\mu \rightarrow e$ conversion is set by SINDRUM II, using a gold target 37 ]

$$
\mathrm{BR}(\mu \rightarrow e)^{\mathrm{Au}}<7 \cdot 10^{-13}
$$


but the future DeeMe [38] and COMET [39] experiments as well as Mu2e [40] aim to improve these bounds using a silicon or an aluminum target. Their projections are

$$
\operatorname{BR}(\mu \rightarrow e)^{\mathrm{Si}}<2 \cdot 10^{-14} \quad \text { and } \quad \operatorname{BR}(\mu \rightarrow e)^{\mathrm{Al}}<6 \cdot 10^{-17} .
$$

The region excluded by the current and future limits are shown in Fig. 9. Compared to the quark flavor constraints for example from meson mixing we see that current lepton flavor constraints are weaker, but will soon become dominant.

$$
\text { Decays } \mu \rightarrow 3 e \text { and } \tau \rightarrow 3 \mu
$$

Finally, we can exploit decays similar to $\mu \rightarrow e \gamma$, but including weak boson effects. The effective Lagrangian parametrizing contributions to decays of the kind $\ell^{\prime} \rightarrow 3 \ell$ can be written as

$$
\mathcal{L}_{\text {eff }}=-2 \sum_{L, R} C_{A B}\left(\bar{\ell}^{\prime} P_{A} \ell\right)\left(\bar{\ell} P_{B} \ell\right),
$$

The corresponding decay width is

$$
\Gamma\left(\ell^{\prime} \rightarrow 3 \ell\right)=\frac{m_{\ell}^{5}}{3 \cdot 2^{12} \pi^{3}}\left(\left|C_{L L}\right|^{2}+\left|C_{R R}\right|^{2}+2\left|C_{L R}^{2}\right|+2\left|C_{R L}\right|^{2}\right) .
$$

Tree-level contributions from flavon exchange are generated from diagrams like the one shown on the right in Fig. 6. The corresponding Wilson coefficients read

$$
C_{L L}=C_{R R}^{*}=g_{\ell \ell^{\prime}}^{*} g_{\ell \ell}^{*}\left(\frac{1}{m_{a}^{2}}-\frac{1}{m_{s}^{2}}\right) \quad \text { and } \quad C_{L R}=C_{R L}^{*}=g_{\ell \ell^{\prime}}^{*} g_{\ell \ell}\left(\frac{1}{m_{a}^{2}}+\frac{1}{m_{s}^{2}}\right) .
$$

In the case of $\mu \rightarrow 3 e$ decays the largest contribution at one loop, as shown on the left of Fig. 6, are suppressed by an additional factor

$$
\frac{\lambda^{2} m_{\tau}}{9 m_{e} m_{\mu}} \approx 0.1
$$

and therefore negligible. For $\tau \rightarrow 3 \ell$ decays, this suppression is even more pronounced.

The most stringent current bounds on flavor violating three-body decays are [41, 42]

$$
\begin{aligned}
& \mathrm{BR}(\tau \rightarrow 3 \mu)<2.1 \cdot 10^{-8} \\
& \operatorname{BR}(\tau \rightarrow 3 e)<2.7 \cdot 10^{-8} \\
& \operatorname{BR}(\mu \rightarrow 3 e)<1.0 \cdot 10^{-12}
\end{aligned}
$$

Mu3e will improve the limit on $\operatorname{BR}(\mu \rightarrow 3 e)$ by at least five orders of magnitude [43]. However, from flavon exchange we only expect branching ratios around $\operatorname{BR}(\mu \rightarrow 3 e)=\mathcal{O}\left(10^{-20}\right), \operatorname{BR}(\tau \rightarrow 3 e)=\mathcal{O}\left(10^{-19}\right)$ and $\operatorname{BR}(\tau \rightarrow 3 e)=\mathcal{O}\left(10^{-16}\right)$. Charged lepton decays with multiple flavor violations such as $\tau \rightarrow \mu e e$ are further phase-space suppressed.

\section{FUTURE HADRON COLLIDER MEASUREMENTS}

Before we discuss the physics opportunities for flavon searches at a $100 \mathrm{TeV}$ hadron collider, we need to briefly consider limits from direct LHC searches. For small flavon masses the main search channel at hadron colliders are the anomalous top decays given in Eq. (24). The current measurement of the total top width at the Tevatron gives $1.10 \mathrm{GeV}<\Gamma_{\text {tot }}<4.05 \mathrm{GeV}$ [44]. The large error bars indicate that this global 

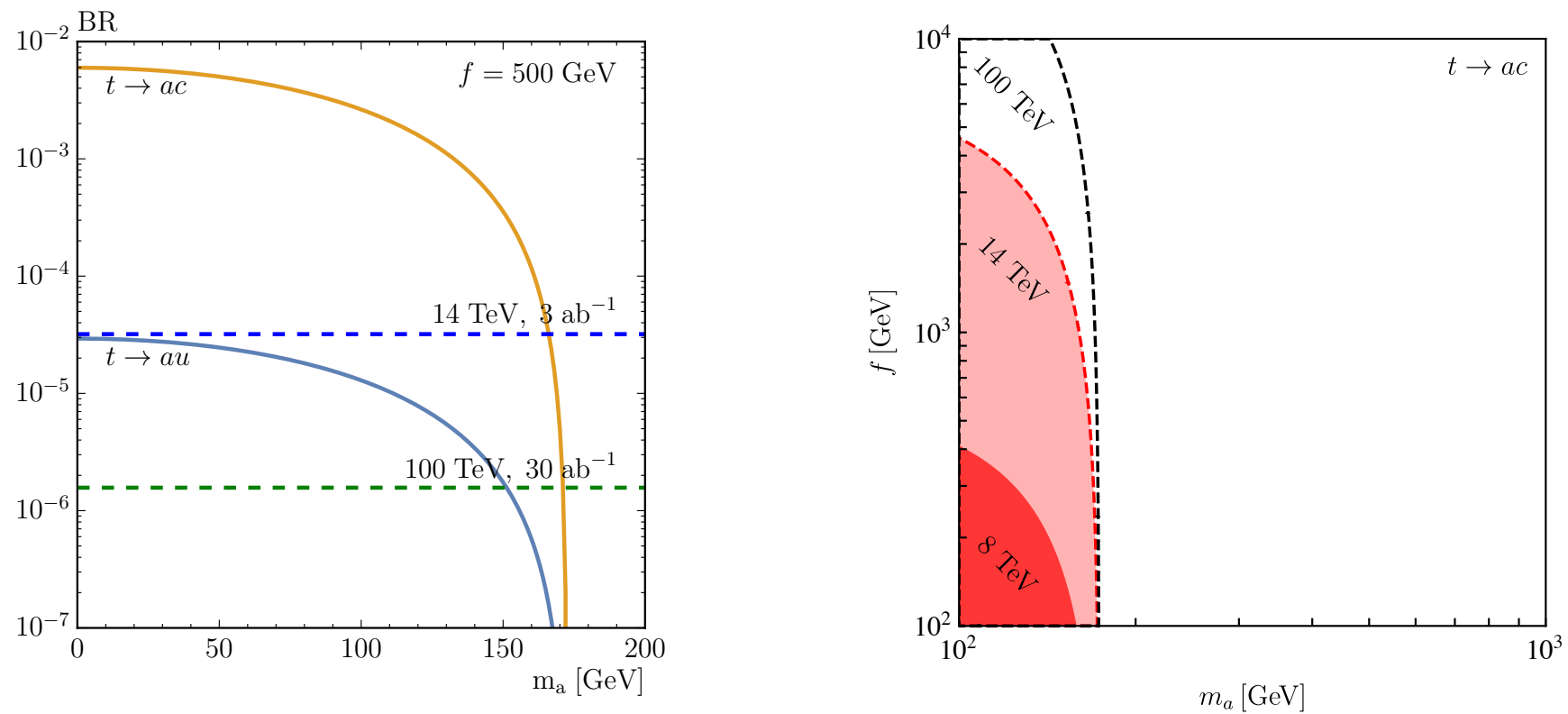

Figure 10. Left: top branching ratios into a flavon and a jet as a function of the flavon mass, assuming a fixed VEV of $f=500 \mathrm{GeV}$. Right: regions in the $m_{a}-f$ plane excluded by these days at the LHC and at a $100 \mathrm{TeV}$ collider.

observable will not help searching for flavon contributions. For the LHC we do not expect this picture to change significantly.

Instead, we can search for specific anomalous decays in analogy to the current limit of $\operatorname{BR}(t \rightarrow H q) \lesssim$ $0.5 \%$ [44. The current and expected reach for such anomalous top decays at the LHC and at a $100 \mathrm{TeV}$ hadron collider is [45]

$$
\begin{aligned}
\mathrm{BR}_{8 \mathrm{TeV}}(t \rightarrow H c) & <5.6 \cdot 10^{-3} \\
\mathrm{BR}_{14 \mathrm{TeV}, 3 \mathrm{ab}^{-1}}(t \rightarrow H c) & <4.5 \cdot 10^{-5} \\
\mathrm{BR}_{100 \mathrm{TeV}, 30 \mathrm{ab}^{-1}}(t \rightarrow H c) & <2.2 \cdot 10^{-6}
\end{aligned}
$$

based on the channel $H \rightarrow b \bar{b}$. Our estimate for a $100 \mathrm{TeV}$ hadron collider comes from scaling the number of expected tops by the leading-order ratio of $\sigma(p p \rightarrow t \bar{t})$ at $14 \mathrm{TeV}$ and $100 \mathrm{TeV}$ with MAdGraph [46]. Assuming a Gaussian scaling the limit of the counting experiment should improve by a factor $6.4 \sqrt{\mathcal{L}_{100 \mathrm{TeV}} / \mathcal{L}_{14} \mathrm{TeV}}$.

We can translate these limits into flavon contributions of the kind $\operatorname{BR}(t \rightarrow a c \rightarrow b \bar{b} c)$, using $\operatorname{BR}(a \rightarrow b \bar{b})>$ $80 \%$ from Fig. 1. We show the expected flavon limits as a function of the flavon mass and couplings in Fig. 10 and find that for a $100 \mathrm{TeV}$ they extend to couplings

$$
\sqrt{g_{i j}^{2}+g_{j i}^{2}} \lesssim \frac{1 \cdot 10^{-3}}{1-\frac{m_{a}}{m_{t}}}
$$

Next, we compute different flavon production rates at hadron colliders. Single flavon production occurs as

$$
g g, b \bar{b} \rightarrow a
$$

where we assume that the collinear bottoms in the final state do not give us an experimental handle on the signal vs background. In addition, there exist associated production channels

$$
b g \rightarrow a b \quad \text { or } \quad u g, c g \rightarrow a t .
$$



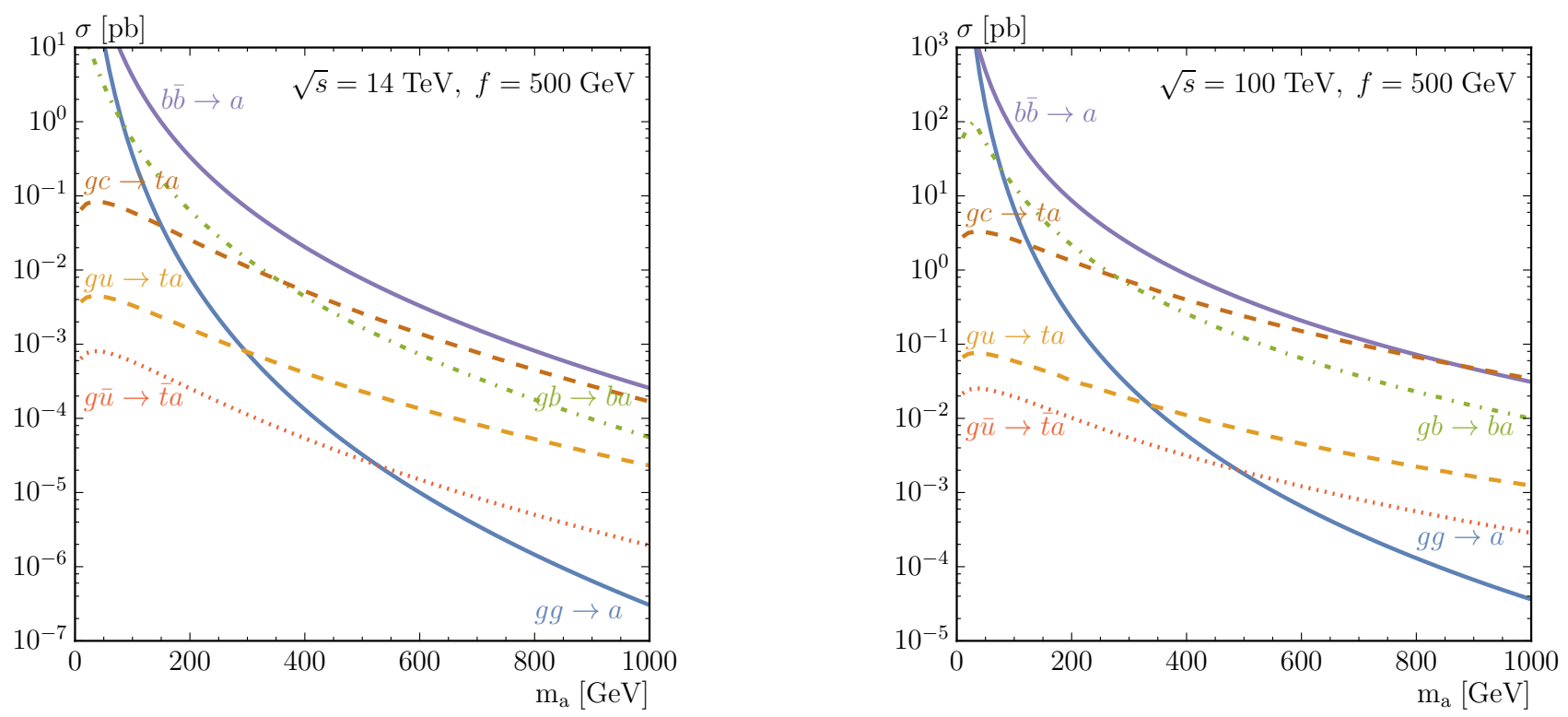

Figure 11. Flavon production cross sections in the different channels for the $14 \mathrm{TeV}$ LHC and a $100 \mathrm{TeV}$ hadron collider using the MSTW2008 PDF set [47]. Couplings are evaluated at $\mu=m_{a}$ or $\mu=m_{a}+m_{t}$ with CRUNDeC [48].

Here, we assume the additional $b$-quark to be hard and central, so it can be tagged. While the bottomassociated channel is driven by a flavor-diagonal coupling $g_{b b}$, the top-associated production indicates a flavor-violating flavon-quark coupling. The different production cross sections for the LHC and for a $100 \mathrm{TeV}$ hadron collider are shown in Fig. 11. At the latter with an integrated luminosity of $30 \mathrm{ab}^{-1}$, we would expect to produce millions of flavons with $m_{a}>500 \mathrm{GeV}$. This leads us to study two kinds of collider signatures:

- first, we can search for traditional resonance decays, like $a \rightarrow \tau \tau$. In that case all production processes in Eqs. (57) and (58) contribute;

- second, we can make use of specific top-associated production, where the flavon decays into $t \bar{q}$ and $\bar{t} q$ for $q=u, c$ are equally likely.

In both cases the key question will be how to control large backgrounds.

\section{Resonance searches}

A direct way to search for a flavon as new dynamical degree of freedom is a resonance search, for example

$$
p p \rightarrow a \rightarrow b \bar{b} / \tau^{+} \tau^{-} .
$$

The $\gamma \gamma$ channel can be discarded, unless we invoke either a diagonal flavon coupling to the tops or a coupling to the $W$-boson. To estimate the discovery potential of a $100 \mathrm{TeV}$ hadron collider, we again scale the current $8 \mathrm{TeV}$ LHC limits assuming Gaussian statistics and an increase of the background cross section by a factor ten. In Tab. I we show some of the $8 \mathrm{TeV}$ limits together with our estimate for a $100 \mathrm{TeV}$ hadron collider. It turns our that only the flavon channel $p p \rightarrow a \rightarrow \tau \tau$ may become sensitive to our benchmark point.

While the above resonances searches are generic for any new (pseudo)scalar, the off-diagonal flavon coupling $g_{t c, c t}$ introduces a single top signature

$$
p p \rightarrow a \rightarrow t \bar{c} / t \bar{u} .
$$




\begin{tabular}{l|cr|cc|cc|cc}
\hline & \multicolumn{2}{|c|}{ ATLAS 8 TeV } & \multicolumn{2}{c|}{ CMS 8 TeV } & \multicolumn{2}{c}{$100 \mathrm{TeV}, 30 \mathrm{ab}^{-1}$} & \multicolumn{2}{c}{ benchmark } \\
$m_{a}[\mathrm{GeV}]$ & 500 & 1000 & 500 & 1000 & 500 & 1000 & 500 & \multicolumn{1}{c}{1000} \\
\hline jet-jet $[\mathrm{pb}]$ & & 0.2 & & & & $2 \cdot 10^{-2}$ & $2.4 \cdot 10^{-2}$ & $1.6 \cdot 10^{-3}$ \\
$\tau^{+} \tau^{-}[\mathrm{pb}]$ & $4 \cdot 10^{-2}$ & $5 \cdot 10^{-3}$ & $4 \cdot 10^{-2}$ & $9 \cdot 10^{-3}$ & $3 \cdot 10^{-3}$ & $4 \cdot 10^{-4}$ & $4.1 \cdot 10^{-3}$ & $3.0 \cdot 10^{-4}$ \\
$\mu^{+} \mu^{-}[\mathrm{pb}]$ & $5 \cdot 10^{-3}$ & $1 \cdot 10^{-3}$ & $2 \cdot 10^{-3}$ & $8 \cdot 10^{-4}$ & $2 \cdot 10^{-4}$ & $6 \cdot 10^{-5}$ & $4.0 \cdot 10^{-5}$ & $2.9 \cdot 10^{-6}$ \\
$\gamma \gamma[\mathrm{pb}]$ & $6 \cdot 10^{-3}$ & $1 \cdot 10^{-3}$ & $2 \cdot 10^{-3}$ & & $2 \cdot 10^{-4}$ & $8 \cdot 10^{-5}$ & $2.3 \cdot 10^{-9}$ & $6.1 \cdot 10^{-11}$ \\
\hline
\end{tabular}

Table I. Current [49 [56] and expected limits for $\sigma \times \mathrm{BR}$ in pb, assuming an increase in the background rate by a factor 10. For the flavon signal we assume $f=500 \mathrm{GeV}$.

The $s$-channel resonance topology only benefits from large branching ratios for heavy flavons, while the $t$ channel topology suffers from two flavon couplings. The SM background is single top production with a NLO cross section $73.5 \mathrm{pb}$ at $100 \mathrm{TeV}$, requiring $\left|\eta_{t}\right|<2.5$ [57. For a flavon with a mass of $500 \mathrm{GeV}$ or $1 \mathrm{TeV}$ we expect for $f=500 \mathrm{GeV} \sigma \times \mathrm{BR}=0.37 \mathrm{pb}$ or $2.9 \cdot 10^{-2} \mathrm{pb}$, respectively. Even before considering the price to pay for charm tagging and without taking into account the top pair background, we note that this channel will obviously not be sensitive.

\section{Associated production}

In addition to these resonance searches, the large flavor-changing coupling $g_{t c, c t}$ allows for top-associated production, Eq. (58). With the relevant flavon decays the collider signatures are

$$
p p \rightarrow t a \rightarrow t b \bar{b} / t \tau^{+} \tau^{-} .
$$

The distinctive case of same-sign top production from the decay $a \rightarrow t \bar{c}$ we will treat below. The decay into bottom quarks suffers from large combinatorial backgrounds and will be overwhelmed by the $t \bar{t}$ background.

A flavon decay to (hadronic) taus can be combined with hadronic top decays, allowing us to reconstruct the final state. The heavy flavon would then decay to two boosted taus, significantly harder than the three top decay jets illustrated in Fig. 12. We start by asking for at least five jets and no isolated leptons,

$$
n_{j} \geq 5 \quad n_{\ell}=0 \quad p_{T, j_{1}}>150 \mathrm{GeV} \quad m_{j_{3} j_{4} j_{5}} \in[140,190] \mathrm{GeV} .
$$

We assume an optimistic $\tau$-tagging efficiency $\epsilon_{\tau}=0.3$ and a misidentification rate of $\epsilon_{j}=10^{-3}$ [58]. To reconstruct the flavon we rely on the collinear approximation in terms of the momentum fractions $x_{1,2}$ of the decaying taus,

$$
m_{\tau \tau}^{2}=\frac{2\left(p_{j_{1}} p_{j_{2}}\right)}{x_{1} x_{2}}
$$

We simulate the flavon signal implemented via FeynRules [59] as well as a fully-hadronic $t \bar{t}$ sample and a $t \bar{t}$ sample with one hadronic top and the other top decaying to a $\tau$-lepton with MADGraPh5+Pythia8 +Delphes3 [46, 60, 61], employing $R=0.4$ anti- $k_{T}$ jets from FASTJet3 [62. For the jets we require $p_{T}>20 \mathrm{GeV}$ and $|\eta|<2.5$. The reconstructed flavon mass distribution for $m_{a}=500 \mathrm{GeV}$ and $f=500 \mathrm{GeV}$ is shown in Fig. 12. It is shifted towards lower masses caused by losses in the reconstruction. The comparison of the expected signal with the background kills any motivation to further study this signature.

\section{Same-sign top pairs}

As alluded to in Eq. (61), the most interesting flavon signature is same-sign top production with an additional jet,

$$
p p \rightarrow t_{\ell} a \rightarrow t_{\ell} t_{\ell} \bar{c}
$$



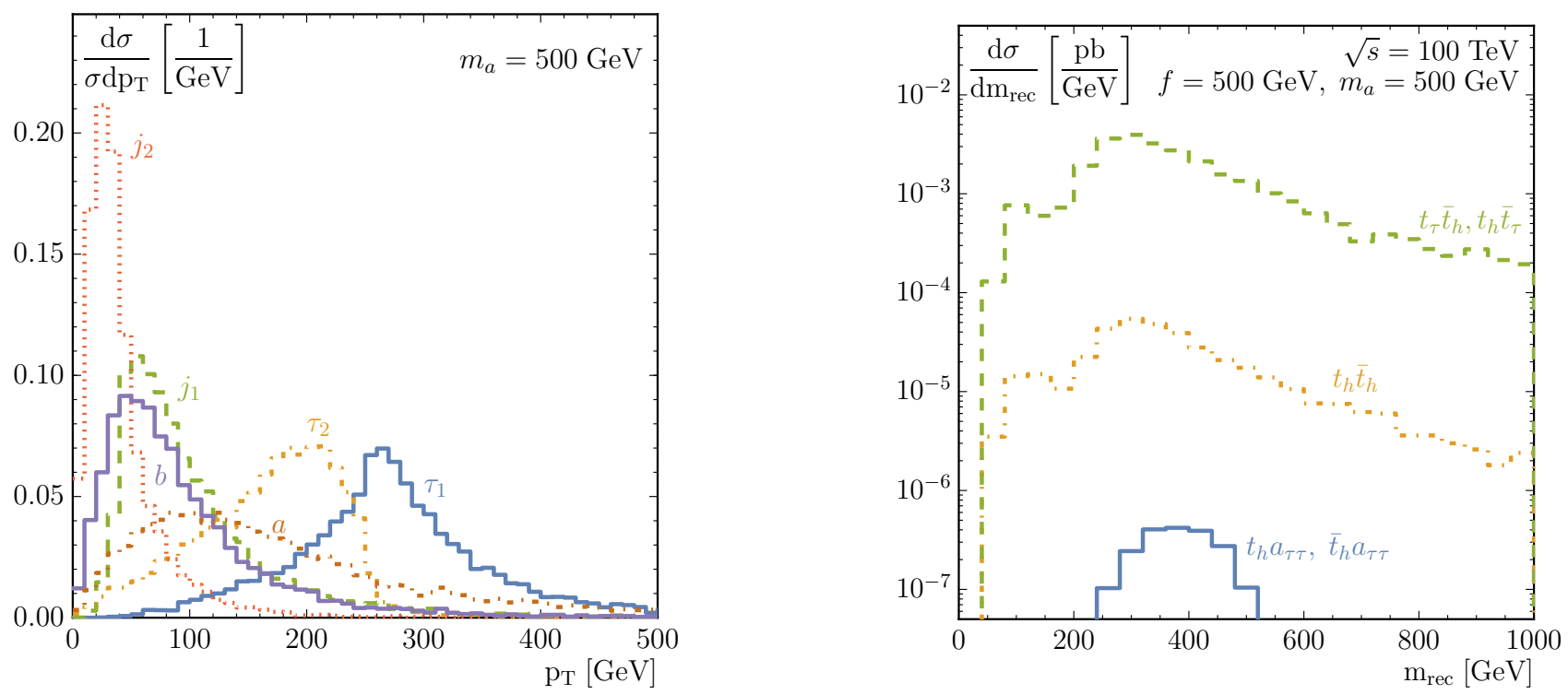

Figure 12. Left: parton-level $p_{T}$ distributions. Right: reconstructed mass distribution. Both figures are simulated for $m_{a}=500 \mathrm{GeV}$.

with a partonic $g c$ initial state. It leads to two same-sign leptons, two $b$-jets, and one additional jet. The $\mathrm{SM}$ background is $p p \rightarrow b b W^{+} W^{+} j$, with a leading order cross section of $5.7 \cdot 10^{-7} \mathrm{pb}$. This means that the irreducible background is actually negligible. Instead, we need to consider consider $t_{\ell} \bar{t} Z j$ and $t_{\ell} \bar{t} W^{+} j$ production, with at least one leptonic top decay and a leptonically decaying weak bosons. We simulate the hard process with MADGRAPH5+Pythia8+Delphes3 [46, 60, 61, 63]. The expected flavon signal has a rate of $5.4 \cdot 10^{-3} \mathrm{pb} \times(500 \mathrm{GeV} / f)^{2}$ for $m_{a}=500 \mathrm{GeV}$. The two leading background are significantly larger, $\sigma_{t_{\ell} \bar{t} W^{+} j}=0.33 \mathrm{pb}$ and $\sigma_{t_{\ell} \bar{t} Z j}=0.48 \mathrm{pb}$.

We require two isolated same-sign leptons with

$$
R_{\text {iso }}=0.2 \quad I_{\text {iso }}=0.1 \quad p_{T, \ell}>10 \mathrm{GeV} \quad\left|\eta_{\ell}\right|<2.5 .
$$

In events with more than two such leptons we pick the hardest two. We veto events with a third lepton of different sign and one opposite-sign combination fulfilling $\left|m_{\ell^{+} \ell^{-}}-m_{Z}\right|<15 \mathrm{GeV}$ to reduce the $t_{\ell} \bar{t} Z j$ background. The hadronic activity is clustered into $R=0.4$ anti- $k_{T}$ jets with $p_{T}>40 \mathrm{GeV}$ and $\left|\eta_{j}\right|<2.5$ using FastJet3 [62]. The hardest jet with $p_{T, j}>100 \mathrm{GeV}$ is our $c$-candidate. Among the non- $c$ jets we require at least two $b$-tags with a parton-level $b$-quark within $R<0.3$ and an assumed tagging efficiency $50 \%$. Finally, we target the two neutrinos by requiring $\not p_{T}>50 \mathrm{GeV}$. This missing transverse momentum has to be distributed between the two branches of the event, the flavon decay and the top decay. A powerful observable for such topologies is $m_{T 2}$ [64]. We define two branches by assigning each $b$-quark to the leptons and minimizing $\Delta R_{\ell_{1} b_{i}}+\Delta R_{\ell_{2} b_{j}}$. Then we assign the hard $c$-jet to the top candidate with the smaller $\Delta y_{(\ell b), j}$. For most signal events we expect $m_{t}<m_{T 2}<m_{a}$, which allows us to search for an excess of events over the background that provides side-bands at high value of $m_{T 2}$. We show the corresponding distribution in the left panel of Fig. 13.

A final, distinctive feature of the signal is that both leptons originate form tops, so the two $b$-jets should be tagged with the same charge [65]. Recent ATLAS studies [66] show that a $b-\bar{b}$ distinction is possible with $\epsilon_{S}=0.2$ and $\epsilon_{B}=0.06$. For our analysis we assume two scenarios: for a conservative estimate we use these ATLAS efficiencies; for a more optimistic case we assume an improved mis-tagging rate of $\epsilon_{B}=0.01$ and an overall $b$-tagging efficiency of $70 \%$. The obtained exclusion limits at $95 \%$ CL with the additional requirement $S / B>0.1$ are illustrated in the right panel of Fig. 13 . 

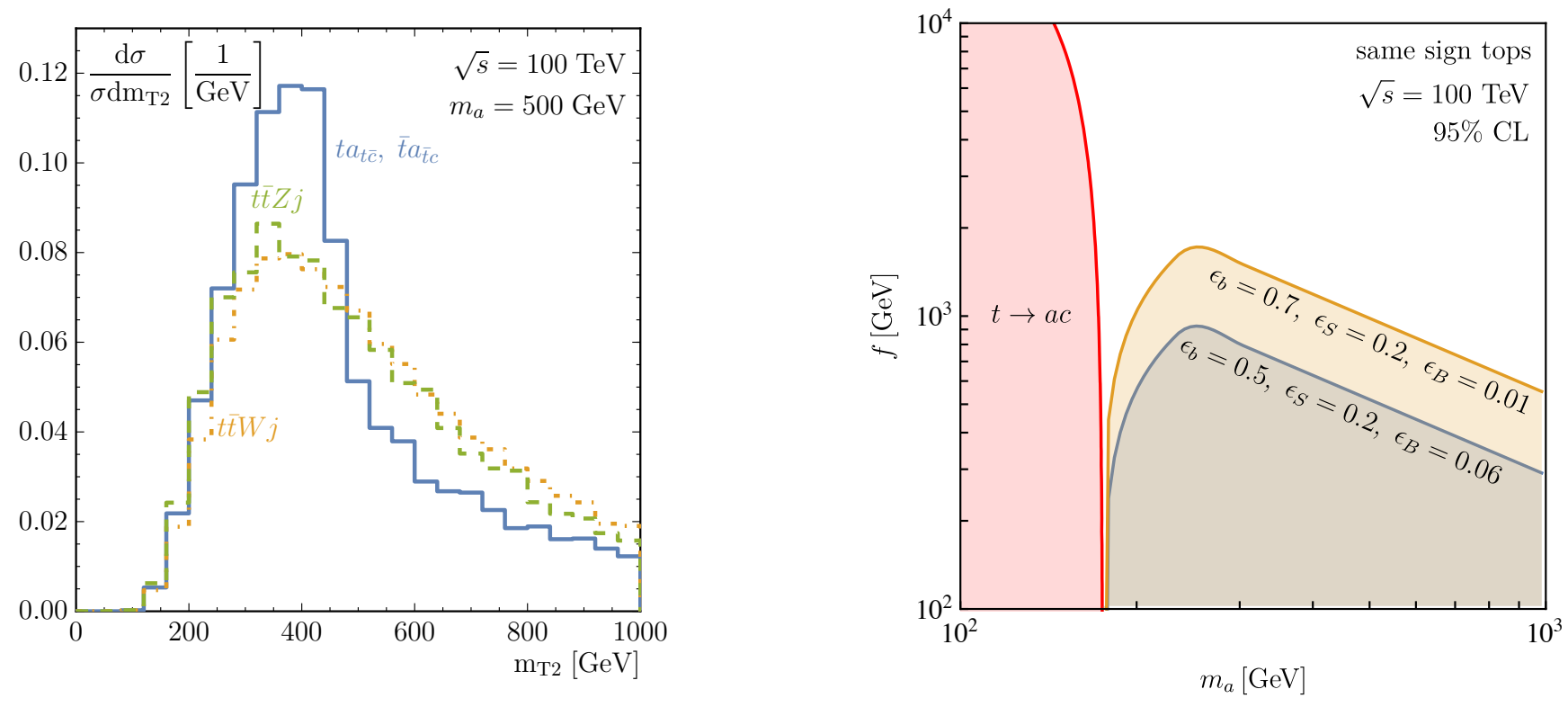

Figure 13. Left: normalized $m_{T 2}$ distribution for a $m_{a}=500 \mathrm{GeV}$ flavon and backgrounds. Right: Exclusion limits from $\sigma(g c \rightarrow t a) \times \mathrm{BR}(a \rightarrow t \bar{c})[\mathrm{pb}]$ at $\sqrt{s}=100 \mathrm{TeV}$. The red area is excluded by $t \rightarrow a c$ decays.

\section{Flavon pair production}

In principle, there is a possibility to study flavon pair production. For the dominant production mode $b \bar{b} \rightarrow f f$ with $g_{a a b b}=2 m_{b} / f^{2}$ and $m_{a}=f=500 \mathrm{GeV}$ we find a production cross section of $1.4 \cdot 10^{-3} \mathrm{pb}$ at $100 \mathrm{TeV}$. The four- $b$ final state will be overwhelmed by combinatorics and QCD backgrounds. The same-sign $t c t c$ channel offers a more distinct signature, but is overwhelmed by a $t \bar{t} W^{+} j j$ cross section of $4.6 \mathrm{pb}$, where we require two jets with $p_{T, j}>100 \mathrm{GeV}$. In addition, this channel would not allow for the reconstruction of a mass peak. Therefore, also flavon pair production can unfortunately be removed from the list of promising discovery channels at a $100 \mathrm{TeV}$ hadron collider.

\section{OUTLOOK}

The experimental consequences of flavor physics models including flavons have been appropriately described in terms of an effective field theory for many decades. In the coming decades we will have the opportunity to test flavon models with a mixed approach of indirect and direct searches, which has been extremely successful in the case of the weak gauge bosons, the top quark, and most recently the Higgs boson.

Starting with current and future quark flavor physics constraints we have shown that a large region in the flavon parameter space is waiting to be probed by alternative experimental approaches in particle physics. While the projected improvements in the quark flavor sector, based on meson mixing and rare decays, are of order-one, lepton flavor experiments should realize their huge potential in the coming years. In Fig. 14 we show how based on our benchmark point the indirect searches for lepton flavor effects will gain immense sensitivity.

In addition to indirect searches in the quark and lepton sectors, systematic direct searches for flavons will for the first time be possible at a $100 \mathrm{TeV}$ hadron collider. Two kinds of collider signatures appear for flavons: first, all colliders search for generic resonant (pseudo-)scalar states, i.e. powerful signatures without a flavon-specific flavor structure. Second, same-sign top pair production with an additional jet coming from the flavon decay $a \rightarrow t j$, combining a distinctive signature with a slightly more background-prone resonance structure. Tools like bottom vs anti-bottom tagging would be extremely useful to extract such signatures at 


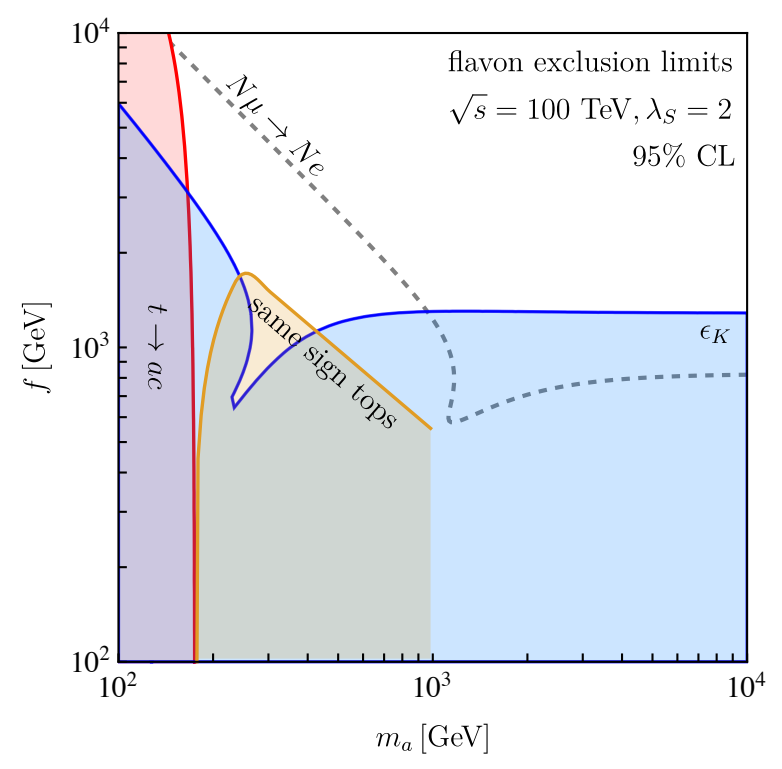

Figure 14. Regions in the $m_{a}-f$ plane which can be probed by quark flavor physics $\left(\epsilon_{K}\right)$, by lepton flavor physics ( $\mu \rightarrow e$ conversion), and by a $100 \mathrm{TeV}$ hadron collider. For the latter we show the reach of anomalous top decays and same-sign top production.

future colliders. In Fig. 14 we show how the projections for a $100 \mathrm{TeV}$ collider nicely add to the indirect searches. Both, quark flavor and lepton flavor searches show distinctive dips close to the diagonal $m_{a} \sim f$, driven by a destructive interference of virtual scalar and pseudo-scalar contributions. On the quark side, anomalous top decays have an excellent coverage for small flavon masses, while the same-sign top channel can cover exactly the weak parts of the indirect searches around $m_{a} \sim f$. In combination, the different tests clearly allow for a systematic and independent coverage of the flavon parameter space in the leptonic sector as well as in the hadronic sector. Ideally this includes a direct discovery of flavon-specific couplings at the $100 \mathrm{TeV}$ hadron collider.

\section{Acknowledgments}

First of all, we would like to thank Jörg Jäckel and Jamie Tattersall for their help in an early phase of the project. We are grateful to Jure Zupan and Andi Weiler for useful discussions regarding flavon pair production. M.B. acknowledges the support of the Alexander von Humboldt Foundation. T.S. would like to thank the International Max Planck Research School for Precision Tests of Fundamental Symmetries for their support. 


\section{Appendix A: Benchmark Point}

To find sample parameter points, we generate random fundamental Yukawa couplings with $y_{i j}^{u, d}=$ $\left|y_{i j}^{u, d}\right| e^{i \phi_{i j}^{u, d}}$ and $\left|y_{i j}^{u, d}\right| \in[0.5,1.5]$ and $\phi_{i j}^{u, d} \in[0,2 \pi]$. The effective Yukawa couplings defined in Eq.(9) have to reproduce the quark and lepton masses, and mixing angles at the flavor breaking scale, which we take to be $1 \mathrm{TeV}$. For the numerical values we use Refs. 67, 68. To this end we perform a $\chi^{2}$ fit, with symmetrized $2 \sigma$ errors and require $\chi^{2}<1 /$ d.o.f.. To illustrate the results in this paper we define a benchmark point with the masses

$$
\begin{aligned}
& m_{u_{i}}=(0.00138,0.563,150.1) \mathrm{GeV} \\
& m_{d_{i}}=(0.00342,0.054,2.29) \mathrm{GeV} \\
& m_{\ell_{i}}=(0.000513,0.106,1.81) \mathrm{GeV} \\
& m_{\nu_{i}}=(0.00161,0.523,3.79) \cdot 10^{-11} \mathrm{GeV},
\end{aligned}
$$

and the mixing matrices

$$
\left|V_{\mathrm{CKM}}\right|=\left(\begin{array}{ccc}
0.974 & 0.226 & 0.0035 \\
0.226 & 0.974 & 0.0388 \\
0.011 & 0.037 & 0.999
\end{array}\right), \quad\left|V_{\mathrm{PMNS}}\right|=\left(\begin{array}{ccc}
0.813 & 0.565 & 0.142 \\
0.483 & 0.519 & 0.705 \\
0.324 & 0.642 & 0.695
\end{array}\right) .
$$

The corresponding Yukawa couplings in the quark sector are

$$
\begin{aligned}
y_{u}= & \left(\begin{array}{ccc}
0.34+0.82 i & -0.23+0.69 i & 0.41-0.43 i \\
-0.84+0.26 i & -0.64+0.32 i & 1.35-0.24 i \\
0.98-0.90 i & -0.84-1.20 i & 0.75+0.65 i
\end{array}\right) \\
y_{d}= & \left(\begin{array}{ccc}
0.53+0.72 i & 0.50-0.34 i & 0.65-0.10 i \\
1.12-0.14 i & 0.93-0.54 i & -0.31-0.65 i \\
-0.16+0.6 i & -0.73+0.34 i & 0.84+0.61 i
\end{array}\right),
\end{aligned}
$$

while the lepton sector is described by

$$
\begin{aligned}
y_{\nu}= & \left(\begin{array}{ccc}
-0.73-0.49 i & 0.91-0.68 i & 0.50-0.21 i \\
0.77+0.36 i & 0.59+0.84 i & 0.23-1.19 i \\
-0.29+1.14 i & -0.02-0.59 i & 1.15+0.91 i
\end{array}\right) \\
y_{\ell}= & \left(\begin{array}{ccc}
0.16+1.29 i & -0.95-0.97 i & 0.25+0.92 i \\
0.008-0.99 i & 1.11+0.40 i & 0.47+0.48 i \\
0.30-1.30 i & 0.22+0.77 i & -0.59-0.018 i
\end{array}\right) .
\end{aligned}
$$

We note that this benchmark point is not optimized to illustrate specific features linked to quark flavor, lepton flavor, and collider reaches. The quark flavor and collider sector on the one hand, and the lepton sector on the other are only loosely related. All couplings are deliberately chosen in the weakly interacting regime, to avoid conclusions too closely tied to assumptions about underlying ultraviolet completions.

[1] C. D. Froggatt and H. B. Nielsen, Nucl. Phys. B 147, 277 (1979).

[2] M. Leurer, Y. Nir and N. Seiberg, Nucl. Phys. B 398, 319 (1993); M. Leurer, Y. Nir and N. Seiberg, Nucl. Phys. B 420, 468 (1994).

[3] H. Georgi and S. L. Glashow, Phys. Rev. D 7, 2457 (1973).

[4] D. B. Kaplan, Nucl. Phys. B 365, 259 (1991).

[5] T. Gherghetta and A. Pomarol, Nucl. Phys. B 586, 141 (2000); Y. Grossman and M. Neubert, Phys. Lett. B 474, 361 (2000); M. Blanke, A. J. Buras, B. Duling, S. Gori and A. Weiler, JHEP 0903, 001 (2009); S. Casagrande, F. Goertz, U. Haisch, M. Neubert and T. Pfoh, JHEP 0810, 094 (2008); M. Bauer, S. Casagrande, U. Haisch and M. Neubert, JHEP 1009, 017 (2010). 
[6] K. Tsumura and L. Velasco-Sevilla, Phys. Rev. D 81, 036012 (2010).

[7] M. Bauer, M. Carena and K. Gemmler, JHEP 1511, 016 (2015); M. Bauer, M. Carena and K. Gemmler, arXiv:1512.03458 [hep-ph].

[8] L. Calibbi, A. Crivellin and B. Zaldovar, Phys. Rev. D 92, no. 1, 016004 (2015).

[9] I. Dorsner and S. M. Barr, Phys. Rev. D 65, 095004 (2002).

[10] A. Ball et.al., Tech. Rep. FCC-ACC-SPC-0001; N. Arkani-Hamed, T. Han, M. Mangano and L. T. Wang, arXiv:1511.06495 [hep-ph].

[11] B. S. Acharya, K. Boek, C. Pongkitivanichkul and K. Sakurai, JHEP 1502, 181 (2015); S. Gori, S. Jung, L. T. Wang and J. D. Wells, JHEP 1412, 108 (2014); J. Bramante, P. J. Fox, A. Martin, B. Ostdiek, T. Plehn, T. Schell and M. Takeuchi, Phys. Rev. D 91, 054015 (2015); A. Berlin, T. Lin, M. Low and L. T. Wang, Phys. Rev. D 91, no. 11, 115002 (2015); S. A. R. Ellis and B. Zheng, Phys. Rev. D 92, no. 7, 075034 (2015); A. Freitas, S. Westhoff and J. Zupan, JHEP 1509, 015 (2015); P. Harris, V. V. Khoze, M. Spannowsky and C. Williams, Phys. Rev. D 93, 054030 (2016); J. Bramante, N. Desai, P. Fox, A. Martin, B. Ostdiek and T. Plehn, arXiv:1510.03460 [hep-ph].

[12] A. J. Barr, M. J. Dolan, C. Englert, D. E. Ferreira de Lima and M. Spannowsky, JHEP 1502, 016 (2015); A. V. Kotwal, S. Chekanov and M. Low, Phys. Rev. D 91, no. 11, 114018 (2015); A. L. Kagan, G. Perez, F. Petriello, Y. Soreq, S. Stoynev and J. Zupan, Phys. Rev. Lett. 114, no. 10, 101802 (2015); M. König and M. Neubert, JHEP 1508, 012 (2015); M. L. Mangano, T. Plehn, P. Reimitz, T. Schell and H. S. Shao, J. Phys. G 43, no. 3, 035001 (2016).

[13] T. G. Rizzo, Phys. Rev. D 89, no. 9, 095022 (2014); T. Cohen, R. T. D’Agnolo, M. Hance, H. K. Lou and J. G. Wacker, JHEP 1411, 021 (2014); A. Hook and A. Katz, JHEP 1409, 175 (2014); C. Borschensky, M. Krmer, A. Kulesza, M. Mangano, S. Padhi, T. Plehn and X. Portell, Eur. Phys. J. C 74, no. 12, 3174 (2014); J. A. AguilarSaavedra, B. Fuks and M. L. Mangano, Phys. Rev. D 91, 094021 (2015); A. J. Larkoski, F. Maltoni and M. Selvaggi, JHEP 1506, 032 (2015); J. Hajer, Y. Y. Li, T. Liu and J. F. H. Shiu, arXiv:1504.07617 [hep-ph]. M. Spannowsky and M. Stoll, arXiv:1505.01921 [hep-ph]; J. Baglio, A. Djouadi and J. Quevillon, arXiv:1511.07853 [hep-ph].

[14] D. Curtin, P. Meade and C. T. Yu, JHEP 1411, 127 (2014).

[15] E. L. Berger, S. B. Giddings, H. Wang and H. Zhang, Phys. Rev. D 90, no. 7, 076004 (2014).

[16] A. J. Buras, F. De Fazio, J. Girrbach, R. Knegjens and M. Nagai, JHEP 1306, 111 (2013).

[17] A. Crivellin, A. Kokulu and C. Greub, Phys. Rev. D 87, no. 9, 094031 (2013).

[18] M. Bona et al. [UTfit Collaboration], JHEP 0803, 049 (2008), http://www.utfit.org/UTfit/

[19] M. Gorbahn, S. Jager, U. Nierste and S. Trine, Phys. Rev. D 84, 034030 (2011).

[20] N. Carrasco et al. [ETM Collaboration], Phys. Rev. D 92, no. 3, 034516 (2015); A. Bazavov et al. [Fermilab Lattice and MILC Collaborations], arXiv:1602.03560 [hep-lat].

[21] J. Charles, S. Descotes-Genon, Z. Ligeti, S. Monteil, M. Papucci and K. Trabelsi, Phys. Rev. D 89, no. 3, 033016 (2014).

[22] A. J. Bevan et al. [UTfit Collaboration], JHEP 1403, 123 (2014).

[23] A. J. Buras, J. Girrbach, D. Guadagnoli and G. Isidori, Eur. Phys. J. C 72, 2172 (2012).

[24] R. Fleischer, arXiv:1212.4967 [hep-ph].

[25] V. Khachatryan et al. [CMS and LHCb Collaborations], Nature 522, 68 (2015).

[26] S. Chatrchyan et al. [CMS Collaboration], Phys. Rev. Lett. 111, 101804 (2013).

[27] R. Aaij et al. [LHCb Collaboration], Phys. Rev. Lett. 111, 101805 (2013).

[28] J. Adam et al. [MEG Collaboration], Phys. Rev. Lett. 110, 201801 (2013).

[29] K. Hayasaka et al. [Belle Collaboration], Phys. Lett. B 666, 16 (2008).

[30] A. M. Baldini et al., arXiv:1301.7225 [physics.ins-det].

[31] R. Harnik, J. Kopp and J. Zupan, JHEP 1303, 026 (2013).

[32] M. A. Shifman, A. I. Vainshtein and V. I. Zakharov, Phys. Lett. B 78, 443 (1978); for a pedagogical discussion of the infamous factor $2 / 27$ see also http://www.thphys.uni-heidelberg.de/ plehn/pics/dark_matter.pdf

[33] A. Crivellin, M. Hoferichter and M. Procura, Phys. Rev. D 89, 054021 (2014).

[34] A. Crivellin, M. Hoferichter and M. Procura, Phys. Rev. D 89, 093024 (2014).

[35] P. Junnarkar and A. Walker-Loud, Phys. Rev. D 87, 114510 (2013).

[36] R. Kitano, M. Koike and Y. Okada, Phys. Rev. D 66, 096002 (2002) [Phys. Rev. D 76, 059902 (2007)]; T. Suzuki, D. F. Measday and J. P. Roalsvig, Phys. Rev. C 35, 2212 (1987).

[37] W. H. Bertl et al. [SINDRUM II Collaboration], Eur. Phys. J. C 47, 337 (2006).

[38] H. Natori [DeeMe Collaboration], Nucl. Phys. Proc. Suppl. 248-250, 52 (2014).

[39] Y. Kuno [COMET Collaboration], PTEP 2013, 022C01 (2013).

[40] R. J. Abrams et al. [Mu2e Collaboration], arXiv:1211.7019 [physics.ins-det].

[41] K. Hayasaka et al., Phys. Lett. B 687, 139 (2010).

[42] U. Bellgardt et al. [SINDRUM Collaboration], Nucl. Phys. B 299, 1 (1988). 
[43] M. Kiehn [Mu3e Collaboration], PoS NUFACT 2014 (2015) 088.

[44] K. A. Olive et al. [Particle Data Group Collaboration], Chin. Phys. C 38, 090001 (2014).

[45] J. A. Aguilar-Saavedra and G. C. Branco, Phys. Lett. B 495, 347 (2000).

[46] J. Alwall et al., JHEP 1407, 079 (2014).

[47] A. D. Martin, W. J. Stirling, R. S. Thorne and G. Watt, Eur. Phys. J. C 63, 189 (2009).

[48] B. Schmidt and M. Steinhauser, Comput. Phys. Commun. 183, 1845 (2012).

[49] G. Aad et al. [ATLAS Collaboration], arXiv:1504.05511 [hep-ex].

[50] G. Aad et al. [ATLAS Collaboration], Phys. Rev. D 90, no. 5, 052005 (2014).

[51] G. Aad et al. [ATLAS Collaboration], JHEP 1507, 157 (2015).

[52] G. Aad et al. [ATLAS Collaboration], Phys. Rev. D 91, no. 5, 052007 (2015).

[53] V. Khachatryan et al. [CMS Collaboration], Phys. Rev. D 91, no. 5, 052009 (2015).

[54] V. Khachatryan et al. [CMS Collaboration], arXiv:1506.02301 [hep-ex].

[55] V. Khachatryan et al. [CMS Collaboration], JHEP 1504, 025 (2015).

[56] CMS Collaboration [CMS Collaboration], CMS-PAS-HIG-14-029.

[57] F. Caola, talk at QCD, EW and Tools at $100 \mathrm{TeV}$, CERN, Oct. 7th 2015, https://indico.cern.ch/event/ 437912/session/2/contribution/11/attachments/1166613/1682282/singletop100TeV.pdf

[58] ATLAS $\tau$-tagging http://cds.cern.ch/record/1562839/files/ATLAS-CONF-2013-064.pdf

[59] A. Alloul, N. D. Christensen, C. Degrande, C. Duhr and B. Fuks, Comput. Phys. Commun. 185, 2250 (2014); N. D. Christensen and C. Duhr, Comput. Phys. Commun. 180, 1614 (2009).

[60] T. Sjöstrand, S. Ask, J. R. Christiansen, R. Corke, N. Desai, P. Ilten, S. Mrenna and S. Prestel et al., Comput. Phys. Commun. 191 (2015) 159.

[61] M. Selvaggi, J. Phys. Conf. Ser. 523 (2014) 012033.

[62] M. Cacciari and G. P. Salam, Phys. Lett. B 641, 57 (2006); M. Cacciari, G. P. Salam and G. Soyez, Eur. Phys. J. C 72, 1896 (2012). http://fastjet.fr

[63] J. Anderson, A. Avetisyan, R. Brock, S. Chekanov, T. Cohen, N. Dhingra, J. Dolen and J. Hirschauer et al., arXiv:1309.1057 [hep-ex]. Detector card adapted for Delphes3.3.

[64] C. G. Lester and D. J. Summers, Phys. Lett. B 463, 99 (1999); A. Barr, C. Lester and P. Stephens, J. Phys. G 29, 2343 (2003).

[65] A. Alves, O. Eboli and T. Plehn, Phys. Rev. D 74, 095010 (2006).

[66] ATLAS Collaboration, ATL-PHYS-PUB-2015-040.

[67] Z. z. Xing, H. Zhang and S. Zhou, Phys. Rev. D 77, 113016 (2008).

[68] M. C. Gonzalez-Garcia, M. Maltoni, J. Salvado and T. Schwetz, JHEP 1212, 123 (2012). 